\title{
Optical electromagnetic radiation density spherical geometric electric and magnetic phase by spherical antiferromagnetic model with fractional system
}

\author{
E. M. Khalii ${ }^{a}$, T. Körpinar ${ }^{b}$, Z. Körpinar ${ }^{c}$, and M. Inc ${ }^{d, e, f, *}$ \\ ${ }^{a}$ Department of Mathematics, College of Science, \\ POBox 11099, Taif University, Taif 21944, Saudi Arabia. \\ ${ }^{b}$ Mus Alparslan University, Department of Mathematics, 49250, Mus, Turkey. \\ ${ }^{c}$ Mus Alparslan University, Department of Administration, 49250 Mus, Turkey. \\ $d$, Department of Computer Engineering, Biruni University, Istanbul, Turkey. \\ e, Furat University, Science Faculty, Department of Mathematics, 23119 Elazig, Turkey. \\ f, Department of Medical Research, China Medical University Hospital, China Medical University, Taichung, Taiwan. \\ *e-mail: minc@firat.edu.tr
}

Received 9 March 2021; accepted 2 April 2021

\begin{abstract}
In this article, we firstly consider a new theory of spherical electromagnetic radiation density with an antiferromagnetic spin of timelike spherical t-magnetic flows by the spherical Sitter frame in de Sitter space. Thus, we construct the new relationship between the new type electric and magnetic phases and spherical timelike magnetic flows de Sitter space $S_{1}^{2}$ Also, we give the applied geometric characterization for spherical electromagnetic radiation density. This concept also boosts to discover of some physical and geometrical characterizations belonging to the particle. Moreover, the solution of the fractional-order systems is considered for the submitted mathematical designs. Graphical demonstrations for fractional solutions are presented to an expression of the approach. The collected results illustrate that mechanism is a relevant and decisive approach to recover numerical solutions of our new fractional equations. Components of performed equations are demonstrated by using approximately explicit values of physical assertions on received solutions. Finally, we construct that electromagnetic fluid propagation along fractional optical fiber indicates a fascinating family of fractional evolution equations with diverse physical and applied geometric modeling in de Sitter space $S_{1}^{2}$.
\end{abstract}

Keywords: t-magnetic particle; optical fiber; geometric phase; evolution equations; traveling wave hypothesis; antiferromagnetic model.

PACS: 02.40.Hw; 03.65.Vf; 05.45.Yv; 03.50.De; 42.15.-i.

MSC 2010: 35C08; 78A05; 53A35.

DOI: https://doi.org/10.31349/RevMexFis.67.050703

\section{Introduction}

An ideal optical fiber has perfect circular symmetry. The polarizations are completely degenerate. Perturbations and imperfections during the fabrication process may introduce anisotropic, which are mostly of a linear or Cartesian type. Sometimes, large linear anisotropic are introduced on purpose, either by modified core geometry or by mechanical stress, to get linear polarizationmaintaining fibers, also called high-birefringence or hi-bi fibers. Bending and squeezing optical fibers does also introduce linear birefringence. Rotational effects of polarization, however, are in general less common and more difficult to produce and to understand.

In recent times, the advancement of glowing lasers and the utilization of optical fiber mechanics have attended to immense importance on flow propagation by curled fluid flows and space-curved. Exclusively torque forces of the geometric phase of isolated light anholonomy with some optical fibers have been investigated; the substance of numerous papers insensitive of their compositions are efficient or analytical. For illustration, Smith [1] examined that the torque of divergence of light generate on monochromatic optical fiber immerse bundle the supervisor is convinced by the electromagnetic fields thanks to the magnetic particle present flows in some optical fiber present transformer. It was still completed that the present is commensurate to torque, which is regular of the order of numerous scopes. Another preliminary substantiation of the geometric ef- fect of the torque of magnetic divergence for light propagating in a magnetic optical fiber detecting a magnetic trajectory was extended [2]. Ross improved a totally geometric system to investigate the reversion in the coiled optical fiber with a fixed-torsion and endorsed its effects with several measurements in the fiber angle into a magnetic fiber. Tomita and Chiao [3] summarized the previous review of Ross for more universal fiber shapes. Also, Chiao and Wu [4] obtained a new important theoretical phase of the results of geometric phase torque. Apart from preceding researches [6-10], we request that a new electromagnetic phase with an antiferromagnetic chain.

Optical fiber investigates, phase is mostly observed by a carrier of new electromagnetic particles and their features. Some nonlinear evolution structures are frequently committed as design to establish substantially complicated scientific developments in diverse provinces of disciplines, particularly in genuine-state physics, chemical physics, plasma physics, optical physics, fluid mechanics, etcetera. Having an advanced perception of the brief substances, likewise their gradual operations in analytical operations and genuine administration in a constructive generation, it is excessively fascinating to satisfaction explicit solutions of approximately systems. Thus there endures no comprehending and unified approach to demonstrate exact solutions of all nonlinear transformation system analysts operate a diversity of diverse concepts [11-23].

It is remarkable that in numerous fields of physics, the utilizations of approximately wave interpretation and its reactions are of 
comprehensive importance. Traveling any wave solutions are a consequential variety of representative with some partial differential aspect and distinct nonlinear fractional differential equations (FDEs) have been established to a selection of traveling some wave improvements. Thus, flood waves are commonly immensely significant of all-instinctive phenomena; they have a superordinary elegant mathematical construction.

Fractional geometry perturbs the operations of derivatives and integrals of optional order. Over the latest several decades, it obtained enormous recognition because of its numerous modeling in distinct scientific competitions. Arbitrary-order designs are softer integer-order designs. FDEs appear in various mathematical and modeling regions comparatively physics, geophysics, polymer rheology, biophysics, capacitor theory, aerodynamics, medicine, nonlinear vibration of earthquake, supervision theory, vital fluid flow phenomena, superelasticity, and magnetical districts. For the intensive study of its utilization, we introduce comprehensive works [24-28].

The outline of the paper is organized as follows: Firstly, we consider a new theory of spherical electromagnetic radiation density with an antiferromagnetic spin of timelike spherical $\mathbf{t}$-magnetic flows by the spherical Sitter frame in de Sitter space. Thus, we construct the new relationship between the new type electric and magnetic phases and spherical timelike magnetic flows de Sitter space $S_{1}^{2}$. Also, we give the applied geometric characterization for spherical electromagnetic radiation density. This concept also boosts to discover of some physical and geometrical characterizations belonging to the particle. Moreover, the solution of the fractionalorder systems is considered for the submitted mathematical designs. Graphical demonstrations for fractional solutions are presented to the expression of the approach. The collected results illustrate that mechanism is a relevant and decisive approach to recover numerical solutions of our new fractional equations. Components of performed equations are demonstrated by using approximately explicit values of physical assertions on received solutions. Finally, we construct that electromagnetic fluid propagation along fractional optical fiber indicates a fascinating family of fractional evolution equations with diverse physical and applied geometric modeling in de Sitter space $S_{1}^{2}$.

\section{Timelike spherical $\mathbf{t}$ - magnetic particle in $S_{1}^{2}$}

In this section, the orthonormal frame design is explained by the orthonormal Lorentzian new spherical Sitter frame and the particle $\gamma: I \rightarrow S_{1}^{2}$ refreshing this spherical Sitter frame equation is defined as Lorentzian spherical particle.

$$
\left[\begin{array}{c}
\nabla_{S} \gamma \\
\nabla_{S} \mathbf{t} \\
\nabla_{s} \mathbf{n}
\end{array}\right]=\left[\begin{array}{lll}
0 & 1 & 0 \\
1 & 0 & \varepsilon \\
0 & \varepsilon & 0
\end{array}\right]\left[\begin{array}{l}
\gamma \\
\mathbf{t} \\
\mathbf{n}
\end{array}\right]
$$

where $\nabla$ is a Levi-Civita form and $\varepsilon=\operatorname{det}(\Psi, \mathbf{t}, \nabla, \mathbf{t})$ is curvature of particle [19]. Then products of spherical vector fields are presented by

$$
\gamma=\mathbf{T} \wedge \mathbf{n}, \quad \mathbf{t}=\gamma \wedge \mathbf{n}, \quad \mathbf{n}=\gamma \wedge \mathbf{T} .
$$

Assume that $\gamma: I \rightarrow S_{1}^{2}$ be a timelike spherical t-magnetic particle and $G$ be the magnetic field in $S_{1}^{2}$ Timelike spherical t-magnetic particle is defined by

$$
\nabla_{S} \mathbf{t}=\phi(\mathbf{t})=G \times \mathbf{t} .
$$

*Lorentz force $\phi$ of a t-magnetic particle with the magnetic field $G$ is presented by

$$
\begin{aligned}
\phi(\gamma) & =\mathbf{t}+\beta \mathbf{n}, \\
\phi(\mathbf{t}) & =\gamma+\varepsilon \mathbf{n}, \\
\phi(\mathbf{n}) & =-\beta \gamma+\varepsilon \mathbf{t}, \\
G^{t} & =\varepsilon \gamma-\beta \mathbf{t}-\mathbf{n},
\end{aligned}
$$

where $\beta=h(\phi(\gamma), \mathbf{n})$.

Putting force equation

$$
m \nabla_{s} \mathbf{t}=q \mathbf{t} \times B+q E,
$$

where

$$
\begin{aligned}
& E=\xi \gamma+\varepsilon \xi \mathbf{n} \\
& \xi=\frac{m+q}{q} .
\end{aligned}
$$

Let $\gamma(s, t)$ is the evolution of $\mathbf{t}$-magnetic particle in de Sitter space. The flow of $\mathbf{t}$-magnetic particle is given by

$$
\nabla_{t} \gamma=\sigma_{1} \mathbf{t}+\sigma_{2} \mathbf{n}
$$

where $\chi_{1}, \chi_{2}$ are potentials.

*Time derivatives of the spherical frame are produced by

$$
\begin{aligned}
& \nabla_{t} \mathbf{t}=\sigma_{1} \gamma+\left(\frac{\partial \sigma_{2}}{\partial s}+\sigma_{1} \varepsilon\right) \mathbf{n}, \\
& \nabla_{t} \gamma=\sigma_{1} \mathbf{t}+\sigma_{2} \mathbf{n} \\
& \nabla_{t} \mathbf{n}=\left(\frac{\partial \sigma_{2}}{\partial s}+\sigma_{1} \varepsilon\right) \mathbf{t}-\sigma_{2} \gamma .
\end{aligned}
$$

* Condition of Lorentz forces $\phi(\gamma), \phi(\mathbf{t}), \phi(\mathbf{n})$ and magnetic field $G^{t}$ for a rotational equilibrium of the timelike t-magnetic particle

$$
\begin{aligned}
\nabla_{t} \phi(\gamma) & =\left(\sigma_{1}-\sigma_{2} \beta\right) \gamma+\beta\left(\frac{\partial \sigma_{2}}{\partial s}+\sigma_{1} \varepsilon\right) \\
& +\left(\left[\frac{\partial \sigma_{2}}{\partial \vartheta}+\varepsilon \sigma_{1}\right]+\frac{\partial \beta}{\partial t}\right) \mathbf{n} \\
\nabla_{t} \phi(\mathbf{t}) & =-\varepsilon \sigma_{2} \gamma+\left(\sigma_{1}+\varepsilon\left[\frac{\partial \sigma_{2}}{\partial s}+\sigma_{1} \varepsilon\right]\right) \mathbf{t}+\left(\frac{\partial \varepsilon}{\partial t}+\sigma_{2}\right) \mathbf{n}, \\
\nabla_{t} \phi(\mathbf{n}) & =\left(\sigma_{1} \varepsilon-\frac{\partial \beta}{\partial t}\right) \gamma+\left(\frac{\partial \varepsilon}{\partial t}-\sigma_{1} \beta\right) \mathbf{t} \\
& +\left(\varepsilon\left[\frac{\partial \sigma_{2}}{\partial s}+\sigma_{1} \varepsilon\right]-\beta \sigma_{2}\right) \mathbf{n}, \\
\nabla_{t} G^{t} & =\left(\frac{\partial \varepsilon}{\partial t}-\beta \sigma_{1}+\sigma_{2}\right) \gamma+\left(\varepsilon \sigma_{1}-\frac{\partial \beta}{\partial t}-\left[\frac{\partial \sigma_{2}}{\partial \vartheta}+\sigma_{1} \varepsilon\right]\right) \mathbf{t} \\
& +\left(\sigma_{2} \varepsilon-\beta\left[\frac{\partial \sigma_{2}}{\partial s}+\sigma_{1} \varepsilon\right]\right) \mathbf{n}, \\
\nabla_{t} E & =-\varepsilon \xi \sigma_{2} \gamma+\left(\xi \sigma_{1}+\varepsilon \xi\left[\frac{\partial \sigma_{2}}{\partial s}+\sigma_{1} \varepsilon\right]\right) \mathbf{t}+\left(\xi \sigma_{2}+\frac{\partial \varepsilon}{\partial t}\right) \mathbf{n},
\end{aligned}
$$

where $\beta=h(\phi(\gamma), \mathbf{n})$. 
${ }^{*}$ In this way, the equation for Lorentz forces $\phi(\gamma), \phi(\mathbf{T}), \phi(\mathbf{N})$ reads

$$
\begin{aligned}
\nabla_{s} \phi(\gamma) & =\gamma+\beta \varepsilon \mathbf{t}+\left(\varepsilon+\frac{\partial \beta}{\partial s}\right) \mathbf{n}, \\
\nabla_{s} \phi(\mathbf{t}) & =\left(1+\varepsilon^{2}\right) \mathbf{t}+\frac{\partial}{\partial s} \varepsilon \mathbf{n}, \\
\nabla_{s} \phi(\mathbf{n}) & =\left(\varepsilon-\frac{\partial \beta}{\partial s}\right) \gamma+\left(\frac{\partial \varepsilon}{\partial s}-\beta\right) \mathbf{t}+\varepsilon^{2} \mathbf{n}, \\
\nabla_{s} G^{T} & =\left(\frac{\partial \varepsilon}{\partial s}-\beta\right) \gamma-\frac{\partial \beta}{\partial s} \mathbf{t}-\beta \varepsilon \mathbf{n},
\end{aligned}
$$

where $\beta=h(\phi(\gamma), \mathbf{n})$.

\section{New geometric results with physical appli- cations}

The geometric density theory, or the theory of density systems, has had and has an enormous impact in applied physical mathematics and an extensive diversity of nonlinear phenomena in mathematical physics. This framework combines questions in nonlinear flux optics, field designs and sigma models, fluid dynamics, relativity, electromagnetic wave theory. In this section, we obtain spherical electric and magnetic radiation density conditions by using Heisenberg antiferromagnetic model.

Spherical electric and magnetic radiation density are given by

$$
\begin{aligned}
& \Phi_{\Pi}^{E^{t}}=\nabla_{t} E^{t} \cdot G^{t} \times \nabla_{t} \Pi, \\
& \Phi_{\Pi}^{G^{t}}=\nabla_{t} G^{t} \cdot G^{t} \times \nabla_{t} \Pi .
\end{aligned}
$$

By using density, a new type of spherical electric and magnetic phase is given by

$$
\Omega_{\Pi}^{E^{t}}=\int\left(\begin{array}{l}
E^{t} \\
\Pi
\end{array}\right) d \varsigma, \quad \Omega_{\Pi}^{G^{t}}=\int\left(\begin{array}{l}
G^{t} \\
\Pi
\end{array}\right) d \varsigma
$$

\section{New type spherical electric and magnetic phase of $\phi(\gamma)$}

Theorem 1. The spherical electric and magnetic phase of $\phi(\gamma)$ are given by

$$
\begin{aligned}
\Omega_{\phi(\mathrm{\Upsilon})}^{\mathrm{E}^{\mathrm{t}}} & =\int\left(-\varepsilon \xi \sigma_{2}\left(\beta\left(\frac{\partial \sigma_{2}}{\partial s}+\sigma_{1} \varepsilon\right)-\beta\left(\left(\frac{\partial \sigma_{2}}{\partial s}+\varepsilon \sigma_{1}\right)+\frac{\partial \beta}{\partial t}\right)\right)-\left(\xi \sigma_{1}+\varepsilon \xi\left(\frac{\partial \sigma_{2}}{\partial s}+\sigma_{1} \varepsilon\right)\right)\left(\varepsilon\left(\left(\frac{\partial \sigma_{2}}{\partial s}+\varepsilon \sigma_{1}\right)+\frac{\partial \beta}{\partial t}\right)\right.\right. \\
& \left.\left.+\left(\sigma_{1}-\sigma_{2} \beta\right)\right)+\left(\xi \sigma_{2}+\frac{\partial \varepsilon}{\partial t} \xi\right)\left(\beta\left(\sigma_{1}-\sigma_{2} \beta\right)+\varepsilon \beta\left(\frac{\partial \sigma_{2}}{\partial s}+\sigma_{1} \varepsilon\right)\right)\right) d \varsigma, \\
\Omega_{\phi(\Upsilon)}^{\mathrm{G}^{\mathrm{t}}} & =\int\left(\left(\frac{\partial \varepsilon}{\partial t}-\beta \sigma_{1}+\sigma_{2}\right)\left(\beta\left(\frac{\partial \sigma_{2}}{\partial s}+\sigma_{1} \varepsilon\right)-\beta\left(\left(\frac{\partial \sigma_{2}}{\partial s}+\varepsilon \sigma_{1}\right)+\frac{\partial \beta}{\partial t}\right)\right)-\left(\varepsilon \sigma_{1}-\frac{\partial \beta}{\partial t}-\left(\frac{\partial \sigma_{2}}{\partial \vartheta}+\sigma_{1} \varepsilon\right)\right)\left(\varepsilon\left(\left(\frac{\partial \sigma_{2}}{\partial s}+\varepsilon \sigma_{1}\right)+\frac{\partial \beta}{\partial t}\right)\right.\right. \\
& \left.\left.+\left(\sigma_{1}-\sigma_{2} \beta\right)\right)+\left(\sigma_{2} \varepsilon-\beta\left(\frac{\partial \sigma_{2}}{\partial s}+\sigma_{1} \varepsilon\right)\right)\left(\beta\left(\sigma_{1}-\sigma_{2} \beta\right)+\varepsilon \beta\left(\frac{\partial \sigma_{2}}{\partial s}+\sigma_{1} \varepsilon\right)\right)\right) d \varsigma .
\end{aligned}
$$

Proof. Definition of magnetic antiferromagnetic spin is given by

$$
\nabla_{t} \phi(\gamma)=\phi(\gamma) \times \nabla_{s} \phi(\gamma)
$$

Then, it is easy to see that

$$
\phi(\gamma) \times \nabla_{s} \phi(\gamma)=\left(\varepsilon+\frac{\partial \beta}{\partial s}-\beta^{2} \varepsilon\right) \gamma-\beta \mathbf{t}-\mathbf{n} .
$$

Straightforward computations lead to

$$
\varepsilon+\frac{\partial \beta}{\partial s}-\beta^{2} \varepsilon=\sigma_{1}-\sigma_{2} \beta, \quad \frac{\partial \sigma_{2}}{\partial s}+\sigma_{1} \varepsilon=-1, \quad\left(\frac{\partial \sigma_{2}}{\partial s}+\varepsilon \sigma_{1}\right)+\frac{\partial \beta}{\partial t}=-1 .
$$

On the other hand, the magnetic-total phase along Lorentz force $\phi(\gamma)$ is indicated by

$$
\Lambda_{\phi(\gamma)}^{G^{t}}=\iint \phi(\Psi) \cdot G^{t} \times \nabla_{t} \phi(\gamma) d \rho
$$

With some calculations, we have

$$
\begin{aligned}
G^{t} \times \nabla_{t} \phi(\gamma) & =\left(\beta\left(\frac{\partial \sigma_{2}}{\partial s}+\sigma_{1} \varepsilon\right)-\beta\left(\left(\frac{\partial \sigma_{2}}{\partial s}+\varepsilon \sigma_{1}\right)+\frac{\partial \beta}{\partial t}\right)\right) \gamma+\left(\varepsilon\left(\left(\frac{\partial \sigma_{2}}{\partial s}+\varepsilon \sigma_{1}\right)+\frac{\partial \beta}{\partial t}\right)\right. \\
& \left.+\left(\sigma_{1}-\sigma_{2} \beta\right)\right) t+\left(\beta\left(\sigma_{1}-\sigma_{2} \beta\right)+\varepsilon \beta\left(\frac{\partial \sigma_{2}}{\partial s}+\sigma_{1} \varepsilon\right)\right) \mathbf{n} .
\end{aligned}
$$

Magnetic anholonomy density of $\phi(\gamma)$ is given by

$$
\delta_{\phi(\gamma)}^{G^{t}}=-\left(\varepsilon\left(\left(\frac{\partial \sigma_{2}}{\partial s}+\varepsilon \sigma_{1}\right)+\frac{\partial \beta}{\partial t}\right)+\left(\sigma_{1}+\sigma_{2} \beta\right)\right)+\beta\left(\beta\left(\sigma_{1}-\sigma_{2} \beta\right)+\varepsilon \beta\left(\frac{\partial \sigma_{2}}{\partial s}+\sigma_{1} \varepsilon\right)\right) .
$$

Also, we present

$$
\Lambda_{\phi(\gamma)}^{G^{t}}=\iint\left(-\left(\varepsilon\left(\left(\frac{\partial \sigma_{2}}{\partial s}+\varepsilon \sigma_{1}\right)+\frac{\partial \beta}{\partial t}\right)+\left(\sigma_{1}+\sigma_{2} \beta\right)\right)+\beta\left(\beta\left(\sigma_{1}-\sigma_{2} \beta\right)+\varepsilon \beta\left(\frac{\partial \sigma_{2}}{\partial s}+\sigma_{1} \varepsilon\right)\right)\right) d \rho .
$$

Spherical electric and magnetic radiation density are given by 


$$
\begin{aligned}
\Phi_{\phi(\Upsilon)}^{\mathrm{E}^{\mathrm{t}}} & =-\varepsilon \xi \sigma_{2}\left(\beta\left(\frac{\partial \sigma_{2}}{\partial s}+\sigma_{1} \varepsilon\right)-\beta\left(\left(\frac{\partial \sigma_{2}}{\partial s}+\varepsilon \sigma_{1}\right)+\frac{\partial \beta}{\partial t}\right)\right)-\left(\xi \sigma_{1}+\varepsilon \xi\left(\frac{\partial \sigma_{2}}{\partial s}+\sigma_{1} \varepsilon\right)\right)\left(\varepsilon\left(\left(\frac{\partial \sigma_{2}}{\partial s}+\varepsilon \sigma_{1}\right)+\frac{\partial \beta}{\partial t}\right)\right. \\
& \left.+\left(\sigma_{1}-\sigma_{2} \beta\right)\right)+\left(\xi \sigma_{2}+\frac{\partial \varepsilon}{\partial t} \xi\right)\left(\beta\left(\sigma_{1}-\sigma_{2} \beta\right)+\varepsilon \beta\left(\frac{\partial \sigma_{2}}{\partial s}+\sigma_{1} \varepsilon\right)\right), \\
\Phi_{\phi(\Upsilon)}^{\mathrm{G}^{\mathrm{t}}} & =\left(\frac{\partial \varepsilon}{\partial t}-\beta \sigma_{1}+\sigma_{2}\right)\left(\beta\left(\frac{\partial \sigma_{2}}{\partial s}+\sigma_{1} \varepsilon\right)-\beta\left(\left(\frac{\partial \sigma_{2}}{\partial s}+\varepsilon \sigma_{1}\right)+\frac{\partial \beta}{\partial t}\right)\right)-\left(\varepsilon \sigma_{1}-\frac{\partial \beta}{\partial t}-\left(\frac{\partial \sigma_{2}}{\partial s}+\sigma_{1} \varepsilon\right)\right)\left(\varepsilon\left(\left(\frac{\partial \sigma_{2}}{\partial s}+\varepsilon \sigma_{1}\right)+\frac{\partial \beta}{\partial t}\right)\right. \\
& \left.+\left(\sigma_{1}-\sigma_{2} \beta\right)\right)+\left(\sigma_{2} \varepsilon-\beta\left(\frac{\partial \sigma_{2}}{\partial s}+\sigma_{1} \varepsilon\right)\right)\left(\beta\left(\sigma_{1}-\sigma_{2} \beta\right)+\varepsilon \beta\left(\frac{\partial \sigma_{2}}{\partial s}+\sigma_{1} \varepsilon\right)\right) .
\end{aligned}
$$

By magnetic anholonomy density with an antiferromagnetic model is obtained

$$
G^{t} \times \phi(\gamma) \times \nabla_{\sigma} \phi(\gamma)=\left(2 \varepsilon+\frac{\partial \beta}{\partial s}-\beta^{2} \varepsilon\right) \mathbf{t}+\beta\left(\frac{\partial \beta}{\partial s}-\beta \varepsilon\right) \mathbf{n} .
$$

After antiferromagnetic condition, we obtain

$$
G^{t A F R} \delta_{\phi(\gamma)}^{G^{t}}=\phi(\gamma) \cdot G^{t} \times \phi(\gamma) \times \nabla_{\sigma} \phi(\gamma)
$$

By this way, we conclude

$$
{ }^{A F R} \delta_{\phi(\gamma)}^{G^{t}}=\left(2 \varepsilon+\frac{\partial \beta}{\partial s}-\beta^{2} \varepsilon\right)+\beta^{2}\left(\frac{\partial \beta}{\partial s}-\beta^{2} \varepsilon\right)
$$

Similarly, we can easily obtain that

$$
{ }^{A F R} \Lambda_{\phi(\gamma)}^{G^{t}}=\iint\left(-\left(2 \varepsilon+\frac{\partial \beta}{\partial s}-\beta^{2} \varepsilon\right)+\beta^{2}\left(\frac{\partial \beta}{\partial s}-\beta^{2} \varepsilon\right)\right) d \rho .
$$

As a reaction, we get the following impressive results.

Theorem 2. Electromagnetic radiation density of $\phi(\gamma)$ is

$$
\begin{aligned}
\mathrm{EMR}_{\phi(\mathrm{Y})}= & \left(\sigma_{2} \varepsilon-\beta\left(\frac{\partial \sigma_{2}}{\partial s}+\sigma_{1} \varepsilon\right)\right)\left(\beta\left(\sigma_{1}-\sigma_{2} \beta\right)+\varepsilon \beta\left(\frac{\partial \sigma_{2}}{\partial s}+\sigma_{1} \varepsilon\right)\right) \\
& -\varepsilon \xi \sigma_{2}\left(\beta\left(\frac{\partial \sigma_{2}}{\partial s}+\sigma_{1} \varepsilon\right)-\beta\left(\left(\frac{\partial \sigma_{2}}{\partial s}+\varepsilon \sigma_{1}\right)+\frac{\partial \beta}{\partial t}\right)\right)-\left(\xi \sigma_{1}+\varepsilon \xi\left(\frac{\partial \sigma_{2}}{\partial s}+\sigma_{1} \varepsilon\right)\right) \\
& \left(\varepsilon\left(\left(\frac{\partial \sigma_{2}}{\partial s}+\varepsilon \sigma_{1}\right)+\frac{\partial \beta}{\partial t}\right)+\left(\sigma_{1}-\sigma_{2} \beta\right)\right)+\left(\xi \sigma_{2}+\frac{\partial \varepsilon}{\partial t} \xi\right)\left(\beta\left(\sigma_{1}-\sigma_{2} \beta\right)\right. \\
& \left.+\varepsilon \beta\left(\frac{\partial \sigma_{2}}{\partial s}+\sigma_{1} \varepsilon\right)\right)+\left(\frac{\partial \varepsilon}{\partial t}-\beta \sigma_{1}+\sigma_{2}\right)\left(\beta\left(\frac{\partial \sigma_{2}}{\partial s}+\sigma_{1} \varepsilon\right)-\beta\left(\left(\frac{\partial \sigma_{2}}{\partial s}+\varepsilon \sigma_{1}\right)+\frac{\partial \beta}{\partial t}\right)\right) \\
& -\left(\varepsilon \sigma_{1}-\frac{\partial \beta}{\partial t}-\left(\frac{\partial \sigma_{2}}{\partial \vartheta}+\sigma_{1} \varepsilon\right)\right)\left(\varepsilon\left(\left(\frac{\partial \sigma_{2}}{\partial s}+\varepsilon \sigma_{1}\right)+\frac{\partial \beta}{\partial t}\right)+\left(\sigma_{1}-\sigma_{2} \beta\right)\right) .
\end{aligned}
$$

Theorem 3. The antiferromagnetic spherical electric and magnetic phase of $\phi(\gamma)$ with antiferromagnetic spin are given by

$$
\begin{aligned}
& { }_{\text {AFR }} \Omega_{\phi(\mathrm{Y})}^{\mathrm{G}^{\mathrm{t}}}=\int\left(-\left(2 \varepsilon+\frac{\partial \beta}{\partial s}-\beta^{2} \varepsilon\right)\left(\varepsilon \sigma_{1}-\frac{\partial \beta}{\partial t}-\left(\frac{\partial \sigma_{2}}{\partial \vartheta}+\sigma_{1} \varepsilon\right)\right)+\left(\sigma_{2} \varepsilon-\beta\left(\frac{\partial \sigma_{2}}{\partial s}+\sigma_{1} \varepsilon\right)\right) \beta\left(\frac{\partial \beta}{\partial s}-\beta^{2} \varepsilon\right)\right) d \varsigma, \\
& { }_{\mathrm{AFR}} \Omega_{\phi(\mathrm{C})}^{\mathrm{E}^{\mathrm{t}}}=\int\left(-\left(\xi \sigma_{1}+\varepsilon \xi\left(\frac{\partial \sigma_{2}}{\partial s}+\sigma_{1} \varepsilon\right)\right)\left(2 \varepsilon+\frac{\partial \beta}{\partial s}-\beta^{2} \varepsilon\right)+\left(\xi \sigma_{2}+\frac{\partial \varepsilon}{\partial t} \xi\right) \beta\left(\frac{\partial \beta}{\partial s}-\beta^{2} \varepsilon\right)\right) d \varsigma .
\end{aligned}
$$

Proof. Spherical electric and magnetic radiation density with an antiferromagnetic model are given by

$$
\begin{aligned}
& { }_{\mathrm{AFR}} \Phi_{\phi(\mathrm{Y})}^{\mathrm{G}^{\mathrm{t}}}=-\left(2 \varepsilon+\frac{\partial \beta}{\partial s}-\beta^{2} \varepsilon\right)\left(\varepsilon \sigma_{1}-\frac{\partial \beta}{\partial t}-\left(\frac{\partial \sigma_{2}}{\partial \vartheta}+\sigma_{1} \varepsilon\right)\right)+\left(\sigma_{2} \varepsilon-\beta\left(\frac{\partial \sigma_{2}}{\partial s}+\sigma_{1} \varepsilon\right)\right) \beta\left(\frac{\partial \beta}{\partial s}-\beta^{2} \varepsilon\right), \\
& { }^{\mathrm{AFR}} \Phi_{\phi(\mathrm{Y})}^{\mathrm{E}^{\mathrm{t}}}=-\left(\xi \sigma_{1}+\varepsilon \xi\left(\frac{\partial \sigma_{2}}{\partial s}+\sigma_{1} \varepsilon\right)\right)\left(2 \varepsilon+\frac{\partial \beta}{\partial s}-\beta^{2} \varepsilon\right)+\left(\xi \sigma_{2}+\frac{\partial \varepsilon}{\partial t} \xi\right) \beta\left(\frac{\partial \beta}{\partial s}-\beta^{2} \varepsilon\right) .
\end{aligned}
$$

If we use above theorem, we may give the following corollary:

Corollary 1. Electromagnetic radiation density with an antiferromagnetic model is

$$
\begin{aligned}
{ }_{\mathrm{AFR}} \Phi_{\phi(\Upsilon)}^{\mathrm{G}^{\mathrm{t}}} & =-\left(2 \varepsilon+\frac{\partial \beta}{\partial s}-\beta^{2} \varepsilon\right)\left(\varepsilon \sigma_{1}-\frac{\partial \beta}{\partial t}-\left(\frac{\partial \sigma_{2}}{\partial \vartheta}+\sigma_{1} \varepsilon\right)\right)+\left(\sigma_{2} \varepsilon-\beta\left(\frac{\partial \sigma_{2}}{\partial s}+\sigma_{1} \varepsilon\right)\right) \beta\left(\frac{\partial \beta}{\partial s}-\beta^{2} \varepsilon\right) \\
& -\left(\xi \sigma_{1}+\varepsilon \xi\left(\frac{\partial \sigma_{2}}{\partial s}+\sigma_{1} \varepsilon\right)\right)\left(2 \varepsilon+\frac{\partial \beta}{\partial s}-\beta^{2} \varepsilon\right)+\left(\xi \sigma_{2}+\frac{\partial \varepsilon}{\partial t} \xi\right) \beta\left(\frac{\partial \beta}{\partial s}-\beta^{2} \varepsilon\right) .
\end{aligned}
$$

Spherical magnetic anholonomy density and time like t-magnetic particle flows in kernel of magnetic quadrupole for timelike spherical evolution with Lorentz force $\phi(\gamma)$. Spherical antiferromagnetic density is provided by the antiferromagnetic density algorithm in Fig. 1 . 


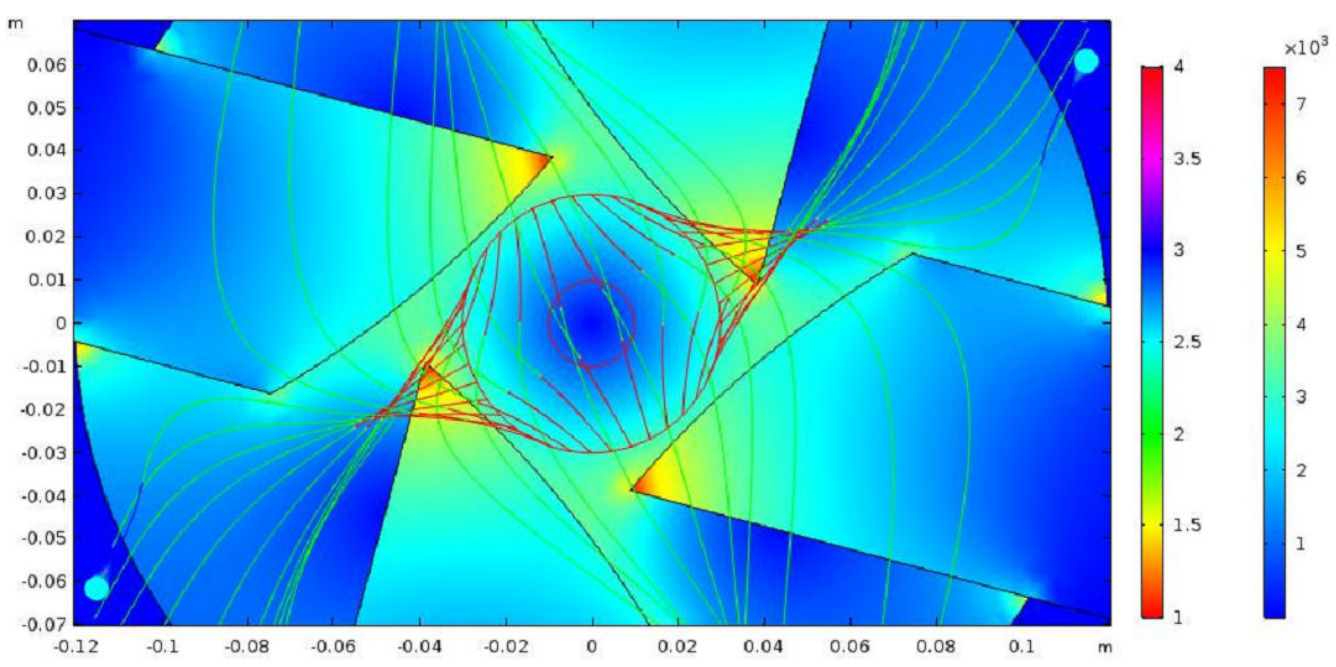

FIGURE 1. Spherical electromagnetic radiation density with $\phi(\mathbf{t})$

\section{Magnetic antiferromagnetic spin for $\phi(t)$}

Theorem 4. Spherical electric and magnetic phase of $\phi(\mathbf{t})$ are given by

$$
\begin{aligned}
\Omega_{\phi(\mathrm{t})}^{\mathrm{G}^{\mathrm{t}}} & =\int\left(\left(\frac{\partial \varepsilon}{\partial t}-\beta \sigma_{1}+\sigma_{2}\right)\left(\sigma_{1}+\varepsilon\left(\frac{\partial \sigma_{2}}{\partial s}+\sigma_{1} \varepsilon\right)-\beta\left(\frac{\partial \varepsilon}{\partial t}+\sigma_{2}\right)\right)-\left(\varepsilon \sigma_{1}-\frac{\partial \beta}{\partial t}-\left(\frac{\partial \sigma_{2}}{\partial \vartheta}+\sigma_{1} \varepsilon\right)\right)\left(\varepsilon\left(\frac{\partial \varepsilon}{\partial t}+\sigma_{2}\right)-\varepsilon \sigma_{2}\right)\right. \\
& \left.+\left(\sigma_{2} \varepsilon-\beta\left(\frac{\partial \sigma_{2}}{\partial s}+\sigma_{1} \varepsilon\right)\right)\left(\varepsilon\left(\sigma_{1}+\varepsilon\left(\frac{\partial \sigma_{2}}{\partial s}+\sigma_{1} \varepsilon\right)\right)-\beta \varepsilon \sigma_{2}\right)\right) d \varsigma, \\
\Omega_{\phi(\mathrm{t})}^{\mathrm{E}^{\mathrm{t}}} & =\int\left(-\left(\sigma_{1}+\varepsilon\left(\frac{\partial \sigma_{2}}{\partial s}+\sigma_{1} \varepsilon\right)-\beta\left(\frac{\partial \varepsilon}{\partial t}+\sigma_{2}\right)\right) \varepsilon \xi \sigma_{2}-\left(\xi \sigma_{1}+\varepsilon \xi\left(\frac{\partial \sigma_{2}}{\partial s}+\sigma_{1} \varepsilon\right)\right)\left(\varepsilon\left(\frac{\partial \varepsilon}{\partial t}+\sigma_{2}\right)-\varepsilon \sigma_{2}\right)\right. \\
& \left.+\left(\xi \sigma_{2}+\frac{\partial \varepsilon}{\partial t} \xi\right)\left(\varepsilon\left(\sigma_{1}+\varepsilon\left(\frac{\partial \sigma_{2}}{\partial s}+\sigma_{1} \varepsilon\right)\right)-\beta \varepsilon \sigma_{2}\right)\right) d \varsigma .
\end{aligned}
$$

Proof. Magnetic antiferromagnetic spin is given by

$$
\nabla_{t} \phi(\mathbf{t})=\phi(\mathbf{t}) \times \nabla_{s} \phi(\mathbf{t})
$$

By definition, we get

$$
\phi(\mathbf{t}) \times \nabla_{s} \phi(\mathbf{t})=-\varepsilon\left(1+\varepsilon^{2}\right) \gamma+\frac{\partial \varepsilon}{\partial s} \mathbf{t}-\left(1-\varepsilon^{2}\right) \mathbf{n} .
$$

Furthermore, we have that

$$
\begin{aligned}
-\varepsilon \sigma_{2} & =-\varepsilon\left(1+\varepsilon^{2}\right), \\
\frac{\partial \varepsilon}{\partial s} & =\sigma_{1}+\varepsilon\left(\frac{\partial \sigma_{2}}{\partial s}+\sigma_{1} \varepsilon\right), \\
\frac{\partial \varepsilon}{\partial t}+\sigma_{2} & =-1-\varepsilon^{2} .
\end{aligned}
$$

The previous system becomes

$$
\begin{aligned}
\sigma_{2} & =\left(1+\varepsilon^{2}\right) \\
\frac{\partial \varepsilon}{\partial s} & =\sigma_{1}+\varepsilon\left(\frac{\partial \sigma_{2}}{\partial s}+\sigma_{1} \varepsilon\right), \\
\frac{\partial \varepsilon}{\partial t} & =-2 \sigma_{2} .
\end{aligned}
$$

Magnetic total spherical phase for force $\phi(\mathbf{t})$ is obtained by

$$
\Lambda_{\phi(\mathbf{t})}^{G^{t}}=\iint \phi(\mathbf{t}) \cdot G^{t} \times \nabla_{t} \phi(\mathbf{t}) d \rho
$$

We can immediately check that 


$$
\begin{aligned}
\Phi_{\phi(\mathbf{t})}^{\mathrm{G}^{\mathrm{t}}} & =\left(\frac{\partial \varepsilon}{\partial t}-\beta \sigma_{1}+\sigma_{2}\right)\left(\sigma_{1}+\varepsilon\left(\frac{\partial \sigma_{2}}{\partial s}+\sigma_{1} \varepsilon\right)-\beta\left(\frac{\partial \varepsilon}{\partial t}+\sigma_{2}\right)\right)-\left(\varepsilon \sigma_{1}-\frac{\partial \beta}{\partial t}\right. \\
& \left.-\left(\frac{\partial \sigma_{2}}{\partial \vartheta}+\sigma_{1} \varepsilon\right)\right)\left(\varepsilon\left(\frac{\partial \varepsilon}{\partial t}+\sigma_{2}\right)-\varepsilon \sigma_{2}\right)+\left(\sigma_{2} \varepsilon-\beta\left(\frac{\partial \sigma_{2}}{\partial s}+\sigma_{1} \varepsilon\right)\right)\left(\varepsilon\left(\sigma_{1}+\varepsilon\left(\frac{\partial \sigma_{2}}{\partial s}+\sigma_{1} \varepsilon\right)\right)-\beta \varepsilon \sigma_{2}\right), \\
\Phi_{\phi(\mathbf{t})}^{\mathrm{E}^{\mathrm{t}}} & =-\left(\sigma_{1}+\varepsilon\left(\frac{\partial \sigma_{2}}{\partial s}+\sigma_{1} \varepsilon\right)-\beta\left(\frac{\partial \varepsilon}{\partial t}+\sigma_{2}\right)\right) \varepsilon \xi \sigma_{2}-\left(\xi \sigma_{1}+\varepsilon \xi\left(\frac{\partial \sigma_{2}}{\partial s}+\sigma_{1} \varepsilon\right)\right)\left(\varepsilon\left(\frac{\partial \varepsilon}{\partial t}+\sigma_{2}\right)-\varepsilon \sigma_{2}\right) \\
& +\left(\xi \sigma_{2}+\frac{\partial \varepsilon}{\partial t} \xi\right)\left(\varepsilon\left(\sigma_{1}+\varepsilon\left(\frac{\partial \sigma_{2}}{\partial s}+\sigma_{1} \varepsilon\right)\right)-\beta \varepsilon \sigma_{2}\right) .
\end{aligned}
$$

Hence, we compute

$$
\left.\delta_{\phi(\mathbf{t})}^{G^{t}}=\left(\sigma_{1}+\varepsilon\left(\frac{\partial \sigma_{2}}{\partial s}+\sigma_{1} \varepsilon\right)-\beta\left(\frac{\partial \varepsilon}{\partial t}+\sigma_{2}\right)\right)+\varepsilon\left(\varepsilon\left(\sigma_{1}+\varepsilon\left(\frac{\partial \sigma_{2}}{\partial s}+\sigma_{1} \varepsilon\right)\right)-\beta \varepsilon \sigma_{2}\right)\right) .
$$

From the magnetic total phase, we obtain

$$
\Lambda_{\phi(\mathbf{t})}^{G^{t}}=\iint\left(\left(\sigma_{1}+\varepsilon\left(\frac{\partial \sigma_{2}}{\partial s}+\sigma_{1} \varepsilon\right)-\beta\left(\frac{\partial \varepsilon}{\partial t}+\sigma_{2}\right)\right)+\varepsilon\left(\varepsilon\left(\sigma_{1}+\varepsilon\left(\frac{\partial \sigma_{2}}{\partial s}+\sigma_{1} \varepsilon\right)\right)-\beta \varepsilon \sigma_{2}\right)\right) d \rho .
$$

It follows that

$$
G^{t} \times \phi(\mathbf{t}) \times \nabla_{s} \phi(\mathbf{t})=\left(\frac{\partial \varepsilon}{\partial s}-\beta\left(1+\varepsilon^{2}\right)\right) \gamma+\left(\varepsilon \frac{\partial \varepsilon}{\partial s}-\varepsilon \beta\left(1+\varepsilon^{2}\right)\right) \mathbf{n} .
$$

Similar to the above, we calculate

$$
A F R \delta_{\phi(t)}^{G^{\Psi}}=\left(\frac{\partial \varepsilon}{\partial s}-\beta\left(1+\varepsilon^{2}\right)\right)+\varepsilon\left(\varepsilon \frac{\partial \varepsilon}{\partial s}-\varepsilon \beta\left(1+\varepsilon^{2}\right)\right)
$$

This implies that

$$
{ }^{A F R} \Lambda_{\phi(t)}^{G^{\Psi}}=\iint\left(\left(\frac{\partial \varepsilon}{\partial s}-\beta\left(1+\varepsilon^{2}\right)\right)+\varepsilon\left(\varepsilon \frac{\partial \varepsilon}{\partial s}-\varepsilon \beta\left(1+\varepsilon^{2}\right)\right)\right) d \rho .
$$

As a reaction, we get the following impressive results.

Theorem 5. The electromagnetic radiation density of $\phi(t)$ is

$$
\begin{aligned}
\mathrm{EMR}_{\phi(\mathbf{t})} & =\left(\sigma_{2} \varepsilon-\beta\left(\frac{\partial \sigma_{2}}{\partial s}+\sigma_{1} \varepsilon\right)\right)\left(\varepsilon\left(\sigma_{1}+\varepsilon\left(\frac{\partial \sigma_{2}}{\partial s}+\sigma_{1} \varepsilon\right)\right)-\beta \varepsilon \sigma_{2}\right)+\left(\frac{\partial \varepsilon}{\partial t}-\beta \sigma_{1}+\sigma_{2}\right)\left(\sigma_{1}+\varepsilon\left(\frac{\partial \sigma_{2}}{\partial s}+\sigma_{1} \varepsilon\right)\right. \\
& \left.-\beta\left(\frac{\partial \varepsilon}{\partial t}+\sigma_{2}\right)\right)-\left(\varepsilon \sigma_{1}-\frac{\partial \beta}{\partial t}-\left(\frac{\partial \sigma_{2}}{\partial \vartheta}+\sigma_{1} \varepsilon\right)\right)\left(\varepsilon\left(\frac{\partial \varepsilon}{\partial t}+\sigma_{2}\right)-\varepsilon \sigma_{2}\right)-\left(\sigma_{1}+\varepsilon\left(\frac{\partial \sigma_{2}}{\partial s}+\sigma_{1} \varepsilon\right)\right. \\
& \left.-\beta\left(\frac{\partial \varepsilon}{\partial t}+\sigma_{2}\right)\right) \varepsilon \xi \sigma_{2}-\left(\xi \sigma_{1}+\varepsilon \xi\left(\frac{\partial \sigma_{2}}{\partial s}+\sigma_{1} \varepsilon\right)\right)\left(\varepsilon\left(\frac{\partial \varepsilon}{\partial t}+\sigma_{2}\right)-\varepsilon \sigma_{2}\right)+\left(\xi \sigma_{2}+\frac{\partial \varepsilon}{\partial t} \xi\right)\left(\varepsilon\left(\sigma_{1}+\varepsilon\left(\frac{\partial \sigma_{2}}{\partial s}+\sigma_{1} \varepsilon\right)\right)-\beta \varepsilon \sigma_{2}\right)
\end{aligned}
$$

Theorem 6. Antiferromagnetic spherical electric and magnetic phase of $\phi(\mathbf{t})$ are given by

$$
\begin{aligned}
& { }^{\mathrm{AFR}} \Omega_{\phi(\mathbf{t})}^{\mathrm{G}^{\mathrm{t}}}=\int\left(\left(\frac{\partial \varepsilon}{\partial t}-\beta \sigma_{1}+\sigma_{2}\right)\left(\frac{\partial \varepsilon}{\partial s}-\beta\left(1+\varepsilon^{2}\right)\right)+\left(\sigma_{2} \varepsilon-\beta\left(\frac{\partial \sigma_{2}}{\partial s}+\sigma_{1} \varepsilon\right)\right)\left(\varepsilon \frac{\partial \varepsilon}{\partial s}-\varepsilon \beta\left(1+\varepsilon^{2}\right)\right)\right) d \varsigma \\
& { }^{\mathrm{AFR}} \Omega_{\phi(\mathbf{t})}^{\mathrm{E}^{\mathrm{t}}}=\int\left(-\left(\frac{\partial \varepsilon}{\partial s}-\beta\left(1+\varepsilon^{2}\right)\right) \varepsilon \xi \sigma_{2}+\left(\varepsilon \frac{\partial \varepsilon}{\partial s}-\varepsilon \beta\left(1+\varepsilon^{2}\right)\right)\left(\xi \sigma_{2}+\frac{\partial \varepsilon}{\partial t} \xi\right)\right) d s .
\end{aligned}
$$

Proof. Spherical electric and magnetic radiation density with an antiferromagnetic model are given by

$$
\begin{aligned}
& { }^{\mathrm{AFR}} \Phi_{\phi(\mathbf{t})}^{\mathrm{G}^{\mathrm{t}}}=\left(\frac{\partial \varepsilon}{\partial t}-\beta \sigma_{1}+\sigma_{2}\right)\left(\frac{\partial \varepsilon}{\partial s}-\beta\left(1+\varepsilon^{2}\right)\right)+\left(\sigma_{2} \varepsilon-\beta\left(\frac{\partial \sigma_{2}}{\partial s}+\sigma_{1} \varepsilon\right)\right)\left(\varepsilon \frac{\partial \varepsilon}{\partial s}-\varepsilon \beta\left(1+\varepsilon^{2}\right)\right), \\
& { }^{\mathrm{AFR}} \Phi_{\phi(\mathrm{t})}^{\mathrm{E}^{\mathrm{t}}}=-\left(\frac{\partial \varepsilon}{\partial s}-\beta\left(1+\varepsilon^{2}\right)\right) \varepsilon \xi \sigma_{2}+\left(\varepsilon \frac{\partial \varepsilon}{\partial s}-\varepsilon \beta\left(1+\varepsilon^{2}\right)\right)\left(\xi \sigma_{2}+\frac{\partial \varepsilon}{\partial t} \xi\right) .
\end{aligned}
$$

Under the antiferromagnetic condition, then we obtain that

$$
G^{t} \times \phi(\mathbf{t}) \times \nabla_{s} \times \phi(\mathbf{t})=\left(\frac{\partial \varepsilon}{\partial s}-\beta\left(1+\varepsilon^{2}\right)\right) \gamma+\left(\varepsilon \frac{\partial \varepsilon}{\partial s}-\varepsilon \beta\left(1+\varepsilon^{2}\right)\right) \mathbf{n}
$$

Corollary 2. Electromagnetic radiation density with an antiferromagnetic model is

$$
\begin{aligned}
\operatorname{AFR}^{\operatorname{EMR}_{\phi(\mathbf{t})}} & =\left(\sigma_{2} \varepsilon-\beta\left(\frac{\partial \sigma_{2}}{\partial s}+\sigma_{1} \varepsilon\right)\right)\left(\varepsilon \frac{\partial \varepsilon}{\partial s}-\varepsilon \beta\left(1+\varepsilon^{2}\right)\right)+\left(\varepsilon \frac{\partial \varepsilon}{\partial s}-\varepsilon \beta\left(1+\varepsilon^{2}\right)\right)\left(\xi \sigma_{2}+\frac{\partial \varepsilon}{\partial t} \xi\right) \\
& +\left(\frac{\partial \varepsilon}{\partial t}-\beta \sigma_{1}+\sigma_{2}\right)\left(\frac{\partial \varepsilon}{\partial s}-\beta\left(1+\varepsilon^{2}\right)\right)-\left(\frac{\partial \varepsilon}{\partial s}-\beta\left(1+\varepsilon^{2}\right)\right) \varepsilon \xi \sigma_{2} .
\end{aligned}
$$

Spherical magnetic anholonomy density and timelike t-magnetic particle flow in the kernel of magnetic quadrupole for timelike spherical evolution with Lorentz force $\phi(\mathbf{t})$. Spherical antiferromagnetic density is provided by the antiferromagnetic density algorithm in Fig. 2. 


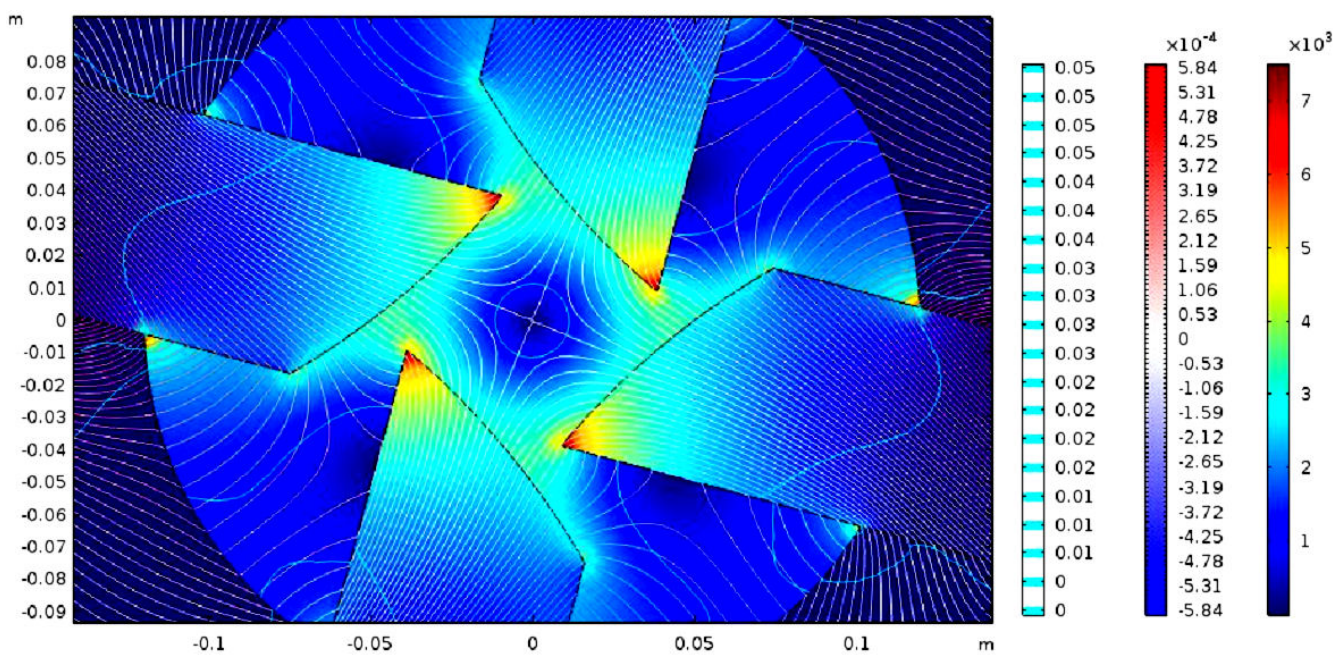

FIGURE 2. Spherical electromagnetic radiation density with $\phi(\mathbf{n})$.

\section{Magnetic antiferromagnetic spin for $\phi(\mathbf{n})$}

Theorem 7. Spherical electric and magnetic phase of $\phi(\mathbf{n})$ are given by

$$
\begin{aligned}
\Omega_{\phi(\mathbf{n})}^{\mathrm{E}^{\mathrm{t}}} & =\int\left(-\varepsilon \xi \sigma_{2}\left(\left(\frac{\partial \varepsilon}{\partial t}-\sigma_{1} \beta\right)-\beta\left(\varepsilon\left(\frac{\partial \sigma_{2}}{\partial s}+\sigma_{1} \varepsilon\right)-\beta \sigma_{2}\right)\right)-\left(\xi \sigma_{1}+\varepsilon \xi\left(\frac{\partial \sigma_{2}}{\partial s}+\sigma_{1} \varepsilon\right)\right)\left(\left(\sigma_{1} \varepsilon-\frac{\partial \beta}{\partial t}\right)\right.\right. \\
& \left.\left.+\varepsilon\left(\varepsilon\left(\frac{\partial \sigma_{2}}{\partial s}+\sigma_{1} \varepsilon\right)-\beta \sigma_{2}\right)\right)+\left(\xi \sigma_{2}+\frac{\partial \varepsilon}{\partial t} \xi\right)\left(\varepsilon\left(\frac{\partial \varepsilon}{\partial t}-\sigma_{1} \beta\right)+\left(\sigma_{1} \varepsilon-\frac{\partial \beta}{\partial t}\right) \beta\right)\right) d \varsigma, \\
\Omega_{\phi(\mathbf{n})}^{\mathrm{G}^{\mathrm{t}}} & =\int\left(\left(\left(\frac{\partial \varepsilon}{\partial t}-\sigma_{1} \beta\right)-\beta\left(\varepsilon\left(\frac{\partial \sigma_{2}}{\partial s}+\sigma_{1} \varepsilon\right)-\beta \sigma_{2}\right)\right)\left(\frac{\partial \varepsilon}{\partial t}-\beta \sigma_{1}+\sigma_{2}\right)-\left(\left(\sigma_{1} \varepsilon-\frac{\partial \beta}{\partial t}\right)+\varepsilon\left(\varepsilon\left(\frac{\partial \sigma_{2}}{\partial s}+\sigma_{1} \varepsilon\right)-\beta \sigma_{2}\right)\right)\right. \\
& \left.\times\left(\varepsilon \sigma_{1}-\frac{\partial \beta}{\partial t}-\left(\frac{\partial \sigma_{2}}{\partial \vartheta}+\sigma_{1} \varepsilon\right)\right)+\left(\sigma_{2} \varepsilon-\beta\left(\frac{\partial \sigma_{2}}{\partial s}+\sigma_{1} \varepsilon\right)\right)\left(\varepsilon\left(\frac{\partial \varepsilon}{\partial t}-\sigma_{1} \beta\right)+\left(\sigma_{1} \varepsilon-\frac{\partial \beta}{\partial t}\right) \beta\right)\right) d \varsigma .
\end{aligned}
$$

Proof. From spherical Sitter frame, we obtain

We instantly calculate

$$
\phi(\mathbf{n}) \times \nabla_{s} \phi(\mathbf{n})=\varepsilon^{3} \gamma-\beta \varepsilon^{2} \mathbf{t}-\left(\left(\varepsilon-\frac{\partial \beta}{\partial s} \varepsilon+\beta\left(\frac{\partial \varepsilon}{\partial s}-\beta\right)\right) \mathbf{n} .\right.
$$

$$
\nabla_{t} \phi(\mathbf{n})=\left(\sigma_{1} \varepsilon-\frac{\partial \beta}{\partial t}\right) \gamma+\left(\frac{\partial \varepsilon}{\partial t}-\sigma_{1} \beta\right) \mathbf{t}+\left(\varepsilon\left(\frac{\partial \sigma_{2}}{\partial s}+\sigma_{1} \varepsilon\right)-\beta \sigma_{2}\right) \mathbf{n} .
$$

Combining the above relations, we get

$$
\sigma_{1} \varepsilon-\frac{\partial \beta}{\partial t}=\varepsilon^{3}, \quad \frac{\partial \varepsilon}{\partial t}-\sigma_{1} \beta=-\beta \varepsilon^{2}, \quad \varepsilon\left(\frac{\partial \sigma_{2}}{\partial s}+\sigma_{1} \varepsilon\right)-\beta \sigma_{2}=\left(\varepsilon-\frac{\partial \beta}{\partial s}\right) \varepsilon+\beta\left(\frac{\partial \varepsilon}{\partial s}-\beta\right) .
$$

Magnetic total spherical phase for spherical Lorentz force $\phi(\mathbf{n})$ is presented by

Consequently, we have

$$
\Phi_{\phi(\mathbf{n})}^{G^{t}}=\iint \phi(\mathbf{n}) \cdot G^{\Psi} \times \nabla_{t} \phi(\mathbf{n}) d \rho .
$$

$$
\begin{aligned}
G^{t} \times \nabla_{t} \phi(\mathbf{n}) & =\left(\left(\frac{\partial \varepsilon}{\partial t}-\sigma_{1} \beta\right)-\beta\left(\varepsilon\left(\frac{\partial \sigma_{2}}{\partial s}+\sigma_{1} \varepsilon\right)-\beta \sigma_{2}\right)\right) \gamma+\left(\left(\sigma_{1} \varepsilon-\frac{\partial \beta}{\partial t}\right)\right. \\
& \left.\left.+\varepsilon\left(\varepsilon\left(\frac{\partial \sigma_{2}}{\partial s}+\sigma_{1} \varepsilon\right)-\beta \sigma_{2}\right)\right) \mathbf{t}+\left(\varepsilon\left(\frac{\partial \sigma_{2}}{\partial s}+\sigma_{1} \varepsilon\right)-\beta \sigma_{2}\right)\right) \mathbf{t}+\left(\varepsilon\left(\frac{\partial \varepsilon}{\partial t}-\sigma_{1} \beta\right)+\left(\sigma_{1} \varepsilon-\frac{\partial \beta}{\partial t}\right) \beta\right) \mathbf{n} .
\end{aligned}
$$

By further calculation, we have that

$$
\begin{aligned}
\Phi_{\phi(\Upsilon)}^{\mathrm{G}^{\mathrm{t}}} & =\left(\left(\frac{\partial \varepsilon}{\partial t}-\sigma_{1} \beta\right)-\beta\left(\varepsilon\left(\frac{\partial \sigma_{2}}{\partial s}+\sigma_{1} \varepsilon\right)-\beta \sigma_{2}\right)\right)\left(\frac{\partial \varepsilon}{\partial t}-\beta \sigma_{1}+\sigma_{2}\right)-\left(\left(\sigma_{1} \varepsilon-\frac{\partial \beta}{\partial t}\right)+\varepsilon\left(\varepsilon\left(\frac{\partial \sigma_{2}}{\partial s}+\sigma_{1} \varepsilon\right)\right.\right. \\
& \left.\left.-\beta \sigma_{2}\right)\right)\left(\varepsilon \sigma_{1}-\frac{\partial \beta}{\partial t}-\left(\frac{\partial \sigma_{2}}{\partial \vartheta}+\sigma_{1} \varepsilon\right)\right)+\left(\sigma_{2} \varepsilon-\beta\left(\frac{\partial \sigma_{2}}{\partial s}+\sigma_{1} \varepsilon\right)\right)\left(\varepsilon\left(\frac{\partial \varepsilon}{\partial t}-\sigma_{1} \beta\right)+\left(\sigma_{1} \varepsilon-\frac{\partial \beta}{\partial t}\right) \beta\right) \\
\Phi_{\phi(\Upsilon)}^{\mathrm{E}^{\mathrm{t}}} & =-\varepsilon \xi \sigma_{2}\left(\left(\frac{\partial \varepsilon}{\partial t}-\sigma_{1} \beta\right)-\beta\left(\varepsilon\left(\frac{\partial \sigma_{2}}{\partial s}+\sigma_{1} \varepsilon\right)-\beta \sigma_{2}\right)\right)-\left(\xi \sigma_{1}+\varepsilon \xi\left(\frac{\partial \sigma_{2}}{\partial s}+\sigma_{1} \varepsilon\right)\right)\left(\left(\sigma_{1} \varepsilon-\frac{\partial \beta}{\partial t}\right)\right. \\
& \left.+\varepsilon\left(\varepsilon\left(\frac{\partial \sigma_{2}}{\partial s}+\sigma_{1} \varepsilon\right)-\beta \sigma_{2}\right)\right)+\left(\xi \sigma_{2}+\frac{\partial \varepsilon}{\partial t} \xi\right)\left(\varepsilon\left(\frac{\partial \varepsilon}{\partial t}-\sigma_{1} \beta\right)+\left(\sigma_{1} \varepsilon-\frac{\partial \beta}{\partial t}\right) \beta\right) .
\end{aligned}
$$


These equations are equivalent to

$$
\delta_{\phi(\mathbf{n})}^{G^{t}}=-\beta\left(\left(\frac{\partial \varepsilon}{\partial t}-\sigma_{1} \beta\right)-\beta\left(\varepsilon\left(\frac{\partial \sigma_{2}}{\partial s}+\sigma_{1} \varepsilon\right)-\beta \sigma_{2}\right)\right)-\varepsilon\left(\left(\sigma_{1} \varepsilon-\frac{\partial \beta}{\partial t}+\varepsilon\left(\varepsilon\left(\frac{\partial \sigma_{2}}{\partial s}+\sigma_{1} \varepsilon\right)-\beta \sigma_{2}\right)\right) .\right.
$$

We immediately compute

$$
\Lambda_{\phi(\mathbf{n})}^{G^{t}}=-\iint\left(-\beta\left(\left(\frac{\partial \varepsilon}{\partial t}-\sigma_{1} \beta\right)-\beta\left(\varepsilon\left(\frac{\partial \sigma_{2}}{\partial s}+\sigma_{1} \varepsilon\right)-\beta \sigma_{2}\right)\right)-\varepsilon\left(\left(\sigma_{1} \varepsilon-\frac{\partial \beta}{\partial t}\right)+\varepsilon\left(\varepsilon\left(\frac{\partial \sigma_{2}}{\partial s}+\sigma_{1} \varepsilon\right)-\beta \sigma_{2}\right)\right)\right) d \rho
$$

By using a spherical Sitter frame, we give

$$
G^{t} \times \phi(\mathbf{n}) \times \nabla_{s} \phi(\mathbf{n})=\left(\beta\left(\left(\varepsilon-\frac{\partial \beta}{\partial s}\right) \varepsilon+\beta\left(\frac{\partial \varepsilon}{\partial s}-\beta\right)\right)-\beta \varepsilon^{2}\right) \gamma+\left(\varepsilon^{3}-\varepsilon\left(\left(\varepsilon-\frac{\partial \beta}{\partial s}\right) \varepsilon+\beta\left(\frac{\partial \varepsilon}{\partial s}-\beta\right)\right)\right) \mathbf{t} .
$$

We immediately compute the density, namely

$$
{ }^{A F R} \delta_{\phi(\mathbf{n})}^{G^{t}}=-\beta\left(\beta\left(\left(\varepsilon-\frac{\partial \beta}{\partial s}\right) \varepsilon+\beta\left(\frac{\partial \varepsilon}{\partial s}-\beta\right)\right)-\beta \varepsilon^{2}\right)-\varepsilon\left(\varepsilon^{3}-\varepsilon\left(\left(\varepsilon-\frac{\partial \beta}{\partial s}\right) \varepsilon+\beta\left(\frac{\partial \varepsilon}{\partial s}-\beta\right)\right)\right) .
$$

Since, we instantly arrive at

$$
{ }^{A F R} \Lambda_{\phi(\mathbf{n})}^{G^{t}}=-\iint\left(\beta\left(\beta\left(\left(\varepsilon-\frac{\partial \beta}{\partial s}\right) \varepsilon+\beta\left(\frac{\partial \varepsilon}{\partial s}-\beta\right)\right)-\beta \varepsilon^{2}\right)+\varepsilon\left(\varepsilon^{3}-\varepsilon\left(\left(\varepsilon-\frac{\partial \beta}{\partial s}\right) \varepsilon+\beta\left(\frac{\partial \varepsilon}{\partial s}-\beta\right)\right)\right) d \rho .\right.
$$

As a reaction, we get the following impressive results.

Theorem 8. The electromagnetic radiation density of $\phi(\mathbf{n})$ is

$$
\begin{aligned}
E M R_{\phi(\mathbf{n})} & =\left(\xi \sigma_{2}+\frac{\partial \varepsilon}{\partial t} \xi\right)\left(\varepsilon\left(\frac{\partial \varepsilon}{\partial t}-\sigma_{1} \beta\right)+\left(\sigma_{1} \varepsilon-\frac{\partial \beta}{\partial t}\right) \beta\right)+\left(\left(\frac{\partial \varepsilon}{\partial t}-\sigma_{1} \beta\right)-\beta\left(\varepsilon\left(\frac{\partial \sigma_{2}}{\partial s}+\sigma_{1} \varepsilon\right)-\beta \sigma_{2}\right)\right)\left(\frac{\partial \varepsilon}{\partial t}-\beta \sigma_{1}+\sigma_{2}\right) \\
& -\left(\left(\sigma_{1} \varepsilon-\frac{\partial \beta}{\partial t}\right)+\varepsilon\left(\varepsilon\left(\frac{\partial \sigma_{2}}{\partial s}+\sigma_{1} \varepsilon\right)-\beta \sigma_{2}\right)\right)\left(\varepsilon \sigma_{1}-\frac{\partial \beta}{\partial t}-\left(\frac{\partial \sigma_{2}}{\partial \vartheta}+\sigma_{1} \varepsilon\right)\right)+\left(\sigma_{2} \varepsilon-\beta\left(\frac{\partial \sigma_{2}}{\partial s}+\sigma_{1} \varepsilon\right)\right)\left(\varepsilon\left(\frac{\partial \varepsilon}{\partial t}-\sigma_{1} \beta\right)\right. \\
& \left.+\left(\sigma_{1} \varepsilon-\frac{\partial \beta}{\partial t}\right) \beta\right)-\varepsilon \xi \sigma_{2}\left(\left(\frac{\partial \varepsilon}{\partial t}-\sigma_{1} \beta\right)-\beta\left(\varepsilon\left(\frac{\partial \sigma_{2}}{\partial s}+\sigma_{1} \varepsilon\right)-\beta \sigma_{2}\right)\right)-\left(\xi \sigma_{1}+\varepsilon \xi\left(\frac{\partial \sigma_{2}}{\partial s}+\sigma_{1} \varepsilon\right)\right) \\
& \times\left(\left(\sigma_{1} \varepsilon-\frac{\partial \beta}{\partial t}+\varepsilon\left(\varepsilon\left(\frac{\partial \sigma_{2}}{\partial s}+\sigma_{1} \varepsilon\right)-\beta \sigma_{2}\right)\right) .\right.
\end{aligned}
$$

Theorem 9. Antiferromagnetic spherical electric and magnetic phase of $\phi(\mathbf{n})$ are given by

$$
\begin{aligned}
{ }^{A F R} \Omega_{\phi(\mathbf{n})}^{G^{t}} & =\int\left(\left(\frac{\partial \varepsilon}{\partial t}-\beta \sigma_{1}+\sigma_{2}\right)\left(\frac{\partial \varepsilon}{\partial s}-\beta\left(1+\varepsilon^{2}\right)\right)+\left(\sigma_{2} \varepsilon-\beta\left(\frac{\partial \sigma_{2}}{\partial s}+\sigma_{1} \varepsilon\right)\right)\left(\varepsilon \frac{\partial \varepsilon}{\partial s}-\varepsilon \beta\left(1+\varepsilon^{2}\right)\right)\right) d \zeta \\
{ }^{A F R} \Omega_{\phi(\mathbf{n})}^{E^{t}} & =\int\left(-\left(\frac{\partial \varepsilon}{\partial s}-\beta\left(1+\varepsilon^{2}\right)\right) \varepsilon \xi \sigma_{2}+\left(\varepsilon \frac{\partial \varepsilon}{\partial s}-\varepsilon \beta\left(1+\varepsilon^{2}\right)\right)\left(\xi \sigma_{2}+\frac{\partial \varepsilon}{\partial t} \xi\right)\right) d \zeta .
\end{aligned}
$$

Proof. By antiferromagnetic model, we get

$$
G^{t} \times \phi(\mathbf{n}) \times \nabla_{s} \phi(\mathbf{n})=\left(\beta\left(\left(\varepsilon-\frac{\partial \beta}{\partial s}\right) \varepsilon+\beta\left(\frac{\partial \varepsilon}{\partial s}-\beta\right)\right)-\beta \varepsilon^{2}\right) \gamma+\left(\varepsilon^{3}-\varepsilon\left(\left(\varepsilon-\frac{\partial \beta}{\partial s}\right) \varepsilon+\beta\left(\frac{\partial \varepsilon}{\partial s}-\beta\right)\right)\right) \mathbf{t} .
$$

Spherical electric and magnetic radiation density with an antiferromagnetic model are given by

$$
\begin{aligned}
& { }^{\mathrm{AFR}} \Phi_{\phi(\mathbf{n})}^{\mathrm{G}^{\mathrm{t}}}=\left(\beta\left(\left(\varepsilon-\frac{\partial \beta}{\partial s}\right) \varepsilon+\beta\left(\frac{\partial \varepsilon}{\partial s}-\beta\right)\right)-\beta \varepsilon^{2}\right)\left(\frac{\partial \varepsilon}{\partial t}-\beta \sigma_{1}+\sigma_{2}\right)-\left(\varepsilon^{3}-\varepsilon\left(\left(\varepsilon-\frac{\partial \beta}{\partial s}\right) \varepsilon+\beta\left(\frac{\partial \varepsilon}{\partial s}-\beta\right)\right)\right)\left(\varepsilon \sigma_{1}-\frac{\partial \beta}{\partial t}-\left(\frac{\partial \sigma_{2}}{\partial \vartheta}+\sigma_{1} \varepsilon\right)\right), \\
& { }^{\mathrm{AFR}} \Phi_{\phi(\mathbf{n})}^{\mathrm{E}^{\mathrm{t}}}=-\varepsilon \xi \sigma_{2}\left(\beta\left(\left(\varepsilon-\frac{\partial \beta}{\partial s}\right) \varepsilon+\beta\left(\frac{\partial \varepsilon}{\partial s}-\beta\right)\right)-\beta \varepsilon^{2}\right)-\left(\xi \sigma_{1}+\varepsilon \xi\left(\frac{\partial \sigma_{2}}{\partial s}+\sigma_{1} \varepsilon\right)\right)\left(\varepsilon^{3}-\varepsilon\left(\left(\varepsilon-\frac{\partial \beta}{\partial s}\right) \varepsilon+\beta\left(\frac{\partial \varepsilon}{\partial s}-\beta\right)\right)\right) .
\end{aligned}
$$

Corollary 1. Electromagnetic radiation density with an antiferromagnetic model is

$$
\begin{aligned}
\mathrm{AFR}^{\mathrm{EMR}_{\phi(\mathbf{n})}} & =-\left(\xi \sigma_{1}+\varepsilon \xi\left(\frac{\partial \sigma_{2}}{\partial s}+\sigma_{1} \varepsilon\right)\right)\left(\varepsilon^{3}-\boldsymbol{\varepsilon}\left(\left(\varepsilon-\frac{\partial \beta}{\partial s}\right) \varepsilon+\beta\left(\frac{\partial \varepsilon}{\partial s}-\beta\right)\right)\right)-\left(\varepsilon^{3}-\varepsilon\left(\left(\varepsilon-\frac{\partial \beta}{\partial s}\right) \varepsilon+\beta\left(\frac{\partial \varepsilon}{\partial s}-\beta\right)\right)\right)\left(\varepsilon \sigma_{1}-\frac{\partial \beta}{\partial t}-\left(\frac{\partial \sigma_{2}}{\partial \vartheta}+\sigma_{1} \varepsilon\right)\right) \\
& +\left(\beta\left(\left(\varepsilon-\frac{\partial \beta}{\partial s}\right) \varepsilon+\beta\left(\frac{\partial \varepsilon}{\partial s}-\beta\right)\right)-\beta \varepsilon^{2}\right)\left(\frac{\partial \varepsilon}{\partial t}-\beta \sigma_{1}+\sigma_{2}\right)-\varepsilon \xi \sigma_{2}\left(\beta\left(\left(\varepsilon-\frac{\partial \beta}{\partial s}\right) \varepsilon+\beta\left(\frac{\partial \varepsilon}{\partial s}-\beta\right)\right)-\beta \varepsilon^{2}\right) .
\end{aligned}
$$

Spherical magnetic anholonomy density and timelike t-magnetic particle flow in the kernel of magnetic quadrupole for timelike spherical evolution with Lorentz force $\phi(\mathbf{n})$. Spherical antiferromagnetic density is provided by antiferromagnetic density algorithm in Fig. 3. 


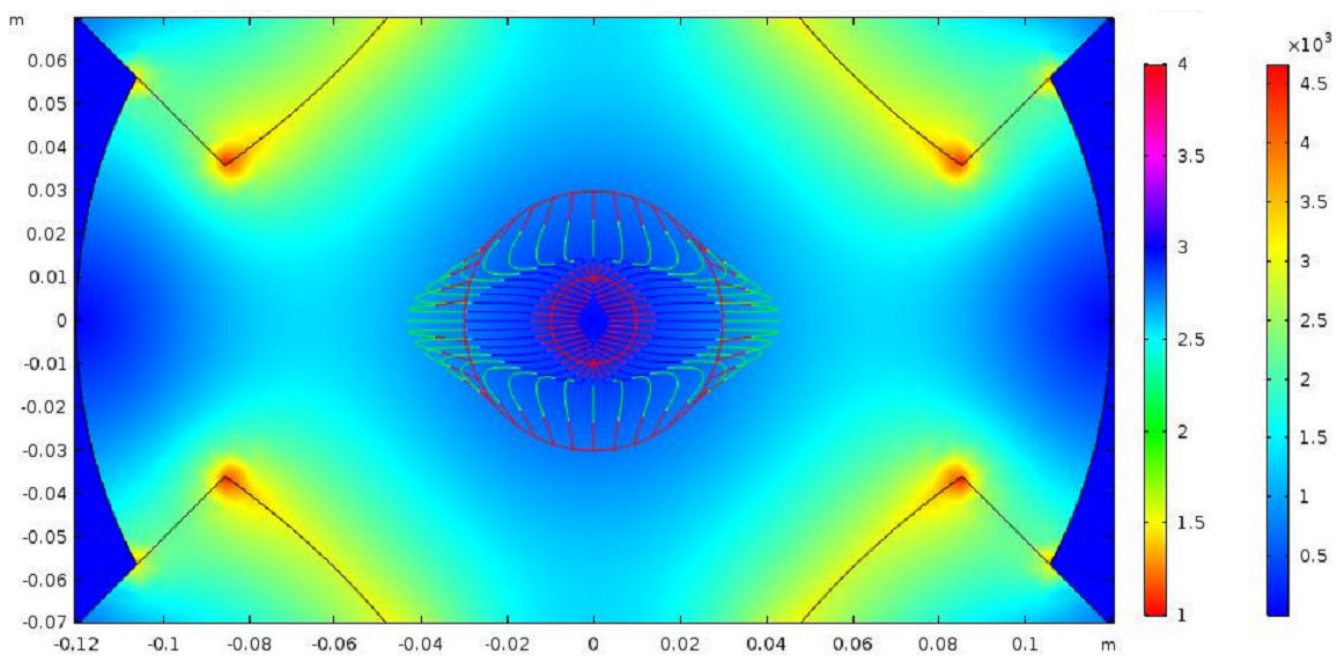

FIGURE 3. Spherical electromagnetic radiation with $\phi(\mathbf{n})$.

\section{Optical Soliton for Extended direct algebraic method with Fractional Equation in $S_{1}^{2}$}

In this section, we design perturbed fractional solutions of the nonlinear evolution equation governing the propagation of solitons by magnetic fields of the polarized light ray traveling in spherical fractional optical fiber. The traveling assumption concept is operated to gauge analytical soliton solutions. Then numerical duplications are also contributed to complement the rational outcomes. Also, we deal with the following evolution equations of Lorentz force $\phi(\mathbf{n})$.

$$
\varepsilon_{t}^{(\eta)}=-2 \sigma_{2}, \quad \varepsilon_{s}=\sigma_{1}+\varepsilon\left(\sigma_{2 s}+\sigma_{1} \varepsilon\right), \quad t>0, \quad 0<\eta \leq 1 .
$$

where $\varepsilon_{t}^{(\eta)}$ is the conformable derivative operator $(\varepsilon=\varepsilon(s, t))$ and $\sigma_{2}=\sigma_{2}(s, t)$. We consider $\sigma_{1}=\sigma_{2}$,

$$
\varepsilon_{t}^{(\eta)}=-2 \sigma_{2}, \quad \varepsilon_{s}=\sigma_{2}+\varepsilon\left(\sigma_{2 s}+\sigma_{2} \varepsilon\right), \quad t>0, \quad 0<\eta \leq 1 .
$$

The conformable derivative of order $\eta \in(0,1]$ is defined as the subsequent interpretation [23]

$$
{ }_{t} D^{\eta} f(t)=\lim _{\vartheta \rightarrow 0} \frac{\zeta\left(t+\vartheta t^{1-\eta}\right)-\zeta(t)}{\vartheta}, \quad \zeta:(0, \infty) \rightarrow R .
$$

Some of the components of conformable derivatives are in such a way $[29,30]$.

$$
\begin{array}{ll}
\text { a) }{ }_{t} D^{\eta} t^{\alpha}=\alpha t^{\alpha-\eta} & \forall \eta \in R, \\
\text { b) }{ }_{t} D^{\eta}(\zeta \chi)=\zeta_{t} D^{\eta} \chi+\chi_{t} D^{\eta} \zeta, & \\
\text { c) }{ }_{t} D^{\eta}(\zeta o \chi)=t^{1-\eta} \chi^{\prime}(t) \zeta^{\prime}(\chi(t)), & \\
\text { d) }{ }_{t} D^{\eta}\left(\frac{\zeta}{\chi}\right)=\frac{\chi_{t} D^{\eta} \zeta-\zeta_{t} D^{\eta} \chi}{\chi^{2}}
\end{array}
$$

- Assume that the traveling wave variable is

$$
\varepsilon(s, t)=u(\phi), \quad \phi=s-Q \frac{t^{\eta}}{\eta}, \quad \sigma_{2}(s, t)=v(\phi),
$$

By using $u(\phi)$ and $v(\phi)$, we have

$$
A\left(u, u_{\phi}, u_{\phi \phi}, u_{\phi \phi \phi}, \ldots\right)=0, \quad B\left(v, v_{\phi}, v_{\phi \phi}, v_{\phi \phi \phi}, \ldots\right)=0 .
$$

- Recognize the solution of Eqs. (4.4),

$$
u(\phi)=\sum_{i=0}^{N} \alpha_{i} G^{i}(\phi), \quad v(\phi)=\sum_{i=0}^{N} \beta_{i} G^{i}(\phi),
$$

where $\alpha_{n} \neq 0, \beta_{n} \neq 0$ and $G(\phi)$ can be defined as:

$$
G^{\prime}(\phi)=\ln (A)\left(f G^{2}(\phi)+\chi G(\phi)+\vartheta\right), \quad A \neq 0,1,
$$

where $\vartheta, \chi, f$ are arbitrary fixeds.

Some solutions of Eq. (4.6) are given by: 
1) If $\chi^{2}-4 \vartheta f<0$ and $f \neq 0$, then

$$
G_{1}(\phi)=-\frac{\chi}{2 f}+\frac{\sqrt{-\left(\chi^{2}-4 \vartheta f\right)}}{2 f} \tan _{A}\left(\frac{\sqrt{-\left(\chi^{2}-4 \vartheta f\right)}}{2} \phi\right), \quad G_{2}(\phi)=-\frac{\chi}{2 f}+\frac{\sqrt{-\left(\chi^{2}-4 \vartheta f\right)}}{2 f} \cot _{A}\left(\frac{\sqrt{-\left(\chi^{2}-4 \vartheta f\right)}}{2} \phi\right)
$$

2) If $\chi^{2}-4 \vartheta f>0$ and $f \neq 0$, then

$$
G_{3}(\phi)=-\frac{\chi}{2 f}+\frac{\sqrt{\chi^{2}-4 \vartheta f}}{4 f}\left(\tanh _{A}\left[\frac{\sqrt{\chi^{2}-4 \vartheta f}}{4} \phi\right]+\operatorname{coth}_{A}\left[\frac{\sqrt{\chi^{2}-4 \vartheta f}}{4} \phi\right]\right) .
$$

3) If $\vartheta f>0$ and $f=0$, then

$$
G_{4}(\phi)=\sqrt{\frac{\vartheta}{f}}\left(\tan _{A}[2 \sqrt{\vartheta f} \phi] \pm \sqrt{\Delta \Omega} \sec _{A}[2 \sqrt{\vartheta f} \phi]\right)
$$

4) If $\vartheta f<0$ and $\chi=0$, then

$$
G_{5}(\phi)=\sqrt{-\frac{\vartheta}{f}}\left(\operatorname{coth}_{A}[2 \sqrt{-\vartheta f} \phi] \pm \sqrt{\Delta \Omega} \operatorname{csch}_{A}[2 \sqrt{-\vartheta f} \phi]\right) .
$$

5) If $\vartheta=f$ and $\chi=0$, then

$$
G_{6}(\phi)=\frac{1}{2}\left(\tan _{A}\left[\frac{\vartheta}{2} \phi\right]-\cot _{A}\left[\frac{\vartheta}{2} \phi\right]\right) .
$$

6) If $\vartheta=-f$ and $\chi=0$, then

$$
G_{7}(\phi)=-\tanh _{A}(\vartheta \phi) .
$$

7) If $\chi^{2}=4 \vartheta f$, then

$$
G_{8}(\phi)=-2 \vartheta \frac{\chi \phi \ln (A)+2}{\chi^{2} \phi \ln (A)} .
$$

8) If $\chi=k, \vartheta=m k,(m \neq 0)$ and $f=0$, then

$$
G_{9}(\phi)=A^{k \phi}-m .
$$

9) If $\chi=f=0$, then

$$
G_{10}(\phi)=\vartheta \phi \ln (A) .
$$

10) If $\chi=\vartheta=0$, then

$$
G_{11}(\phi)=-\frac{1}{f \phi \ln (A)} .
$$

11) If $\vartheta=0$, and $\chi \neq 0$, then

$$
G_{12}(\phi)=-\frac{\Delta \chi}{f\left(\cosh _{A}(\chi \phi)-\sinh _{A}(\chi \phi)+\Delta\right)} .
$$

12) If $\chi=k, \vartheta=0$ and $f=m k(m \neq 0)$, then

$$
G_{13}(\phi)=\frac{\Delta A^{k \phi}}{\Omega-m \Delta A^{k \phi}} .
$$


Remark. The generalized hyperbolic and triangular functions are defined [29];

$$
\begin{aligned}
\sin _{A}(\phi) & =\frac{\Delta A^{i \phi}-\Omega A^{-i \phi}}{2 i}, & \cos A & =\frac{\Delta A^{i \phi}+\Omega A^{-i \phi}}{2}, \\
\tan _{A}(\phi) & =-i \frac{\Delta A^{i \phi}-\Omega A^{-i \phi}}{\Delta A^{i \phi}+\Omega A^{-i \phi}}, & \cot _{A}(\phi) & =i \frac{\Delta A^{i \phi}+\Omega A^{-i \phi}}{\Delta A^{i \phi}-\Omega A^{-i \phi}}, \\
\sec _{A}(\phi) & =\frac{2}{\Delta A^{i \phi}+\Omega A^{-i \phi}}, & \csc _{A}(\phi) & =\frac{2 i}{\Delta A^{i \phi}-\Omega A^{-i \phi}}, \\
\sinh _{A}(\phi) & =\frac{\Delta A^{\phi}-\Omega A^{-\phi}}{2}, & \cosh _{A}(\phi) & =\frac{\Delta A^{\phi}+\Omega A^{-\phi}}{2}, \\
\tanh _{A}(\phi) & =\frac{\Delta A^{\phi}-\Omega A^{-\phi}}{\Delta A^{\phi}+\Omega A^{-\phi}}, & \operatorname{coth}_{A}(\phi) & =\frac{\Delta A^{\phi}+\Omega A^{-\phi}}{\Delta A^{\phi}-\Omega A^{-\phi}}, \\
\operatorname{sech}_{A}(\phi) & =\frac{2}{\Delta A^{\phi}+\Omega A^{-\phi}}, & \operatorname{csch}_{A}(\phi) & =\frac{2}{\Delta A^{\phi}-\Omega A^{-\phi}},
\end{aligned}
$$

By placing the above equations, we have

$$
\begin{array}{r}
2 v(\phi)-Q u^{\prime}(\phi)=0 \\
u^{\prime}(\phi)-u(\phi) v^{\prime}(\phi)-v(\phi) u(\phi)^{2}-v(\phi)=0
\end{array}
$$

The solutions of the above equation be a finite series as follows:

$$
u(\phi)=\sum_{j=0}^{N} \alpha_{j} G^{j}(\phi), \quad v(\phi)=\sum_{j=0}^{M} \beta_{j} G^{j}(\phi),
$$

where $G(\phi)$ satisfies Eq. (4.6) $\phi=\sigma-Q\left(t^{\eta} / \eta\right)$ and $\alpha_{j}, \beta_{j}$ for $j=\overline{1, N}$ are values to be definited.

Putting

$$
u(\phi)=\alpha_{0}+\alpha_{1} G(\phi), \quad v(\phi)=\beta_{0}+\beta_{1} G(\phi)+\beta_{2} G(\phi)^{2},
$$

where $G(\phi)$ satisfied Eq. (4.6).

Also, solving the above system, we get

$$
\alpha_{0}=-\chi \ln (A), \quad \alpha_{1}=-2 f \ln (A), \quad \beta_{0}=-2 f \vartheta \ln (A)^{2}, \quad \beta_{1}=-2 f \chi \ln (A)^{2}, \beta_{2}=-2 f^{2} \ln (A)^{2}, \quad Q=2 .
$$

New solutions of Eq. (4.1) are presented by:

1) If $\chi^{2}-4 \vartheta f<0$ and $f \neq 0$, then

$$
\begin{aligned}
\varepsilon_{1}(s, t) & =-\chi \ln (A)-2 f \ln (A)\left(-\frac{\chi}{2 f}+\frac{\sqrt{-\left(\chi^{2}-4 \vartheta f\right)}}{2 f} \tan _{A}\left[\frac{\sqrt{-\left(\chi^{2}-4 \vartheta f\right)}}{2} \phi\right]\right) \\
\sigma_{21}(s, t) & =-2 f \vartheta \ln (A)^{2}-2 f \chi \ln (A)^{2}\left(-\frac{\chi}{2 f}+\frac{\sqrt{-\left(\chi^{2}-4 \vartheta h f\right)}}{2 f} \tan _{A}\left[\frac{\sqrt{-\left(\chi^{2}-4 \vartheta f\right)}}{2} \phi\right]\right) \\
& -2 f^{2} \ln (A)^{2}\left(-\frac{\chi}{2 f}+\frac{\sqrt{-\left(\chi^{2}-4 \vartheta f\right)}}{2 f} \tan _{A}\left[\frac{\sqrt{-\left(\chi^{2}-4 \vartheta f\right)}}{2} \phi\right]\right)^{2} \\
\varepsilon_{2}(s, t) & =-\chi \ln (A)-2 f \ln (A)\left(-\frac{\chi}{2 f}+\frac{\sqrt{-\left(\chi^{2}-4 \vartheta f\right)}}{2 f} \cot _{A}\left[\frac{\sqrt{-\left(\chi^{2}-4 \vartheta f\right)}}{2} \phi\right]\right) \\
\sigma_{22}(s, t) & =-2 f \vartheta \ln (A)^{2}-2 f \chi \ln (A)^{2}\left(-\frac{\chi}{2 f}+\frac{\sqrt{-\left(\chi^{2}-4 \vartheta f\right)}}{2 f} \cot _{A}\left[\frac{\sqrt{-\left(\chi^{2}-4 \vartheta f\right)}}{2} \phi\right]\right) \\
& -2 f^{2} \ln (A)^{2}\left(-\frac{\chi}{2 f}+\frac{\sqrt{-\left(\chi^{2}-4 \vartheta f\right)}}{2 f} \cot _{A}\left[\frac{\sqrt{-\left(\chi^{2}-4 \vartheta f\right)}}{2} \phi\right)^{2} \cdot\right.
\end{aligned}
$$

2) If $\chi^{2}-4 \vartheta f>0$ and $f \neq 0$, then singular soliton and dark solutions are presented by 


$$
\begin{aligned}
\varepsilon_{3}(s, t) & =-\chi \ln (A)-2 f \ln (A)\left(-\frac{\chi}{2 f}+\frac{\sqrt{\chi^{2}-4 \vartheta f}}{4 f}\left[\tanh _{A}\left\{\frac{\sqrt{\chi^{2}-4 \vartheta f}}{4} \phi\right\}+\operatorname{coth}_{A}\left\{\frac{\sqrt{\chi^{2}-4 \vartheta f}}{4} \phi\right\}\right]\right), \\
\sigma_{23}(s, t) & =-2 f \vartheta \ln (A)^{2}-2 f \chi \ln (A)^{2}\left(-\frac{\chi}{2 f}+\frac{\sqrt{\chi^{2}-4 \vartheta f}}{4 f}\left[\tanh _{A}\left\{\frac{\sqrt{\chi^{2}-4 \vartheta f}}{4} \phi\right\}+\operatorname{coth}_{A}\left\{\frac{\sqrt{\chi^{2}-4 \vartheta f}}{4} \phi\right\}\right]\right) \\
& -2 f^{2} \ln (A)^{2}\left(-\frac{\chi}{2 f}+\frac{\sqrt{\chi^{2}-4 \vartheta f}}{4 f}\left[\tanh _{A}\left\{\frac{\sqrt{\chi^{2}-4 \vartheta f}}{4} \phi\right\}+\operatorname{coth}_{A}\left\{\frac{\sqrt{\chi^{2}-4 \vartheta f}}{4} \phi\right\}\right]\right)^{2} .
\end{aligned}
$$

3) If $\vartheta f>0$ and $\chi=0$, then the singular new periodic solutions are presented by

$$
\begin{aligned}
\varepsilon_{4}(s, t) & =-g \ln (A)-2 f \ln (A)\left(\sqrt{\frac{\vartheta}{f}}\left[\tan _{A}\{2 \sqrt{\vartheta f} \phi\} \pm \sqrt{\Delta \Omega} \sec _{A}\{2 \sqrt{\vartheta f} \phi\}\right]\right) \\
\sigma_{24}(s, t) & =-2 f \vartheta \ln (A)^{2}+(-2) f \chi \ln (A)^{2}\left(\sqrt{\frac{\vartheta}{f}}\left[\tan _{A}\{2 \sqrt{\vartheta f} \phi\} \pm \sqrt{\Delta \Omega} \sec _{A}\{2 \sqrt{\vartheta f} \phi\}\right]\right) \\
& -2 f^{2} \ln (A)^{2}\left(\sqrt{\frac{\vartheta}{f}}\left[\tan _{A}\{2 \sqrt{\vartheta f} \phi\} \pm \sqrt{\Delta \Omega} \sec _{A}\{2 \sqrt{\vartheta f} \phi\}\right]\right)^{2} .
\end{aligned}
$$

4) If $\vartheta f<0$ and $\chi=0$, then the dark, bright, and singular soliton solutions are presented by

$$
\begin{aligned}
\varepsilon_{5}(s, t) & =-\chi \ln (A)-2 f \ln (A)\left(\sqrt{-\frac{\vartheta}{f}}\left[-\operatorname{coth}_{A}\{2 \sqrt{-\vartheta f} \phi\} \pm \sqrt{\Delta \Omega} \operatorname{csch}_{A}\{2 \sqrt{-\vartheta f} \phi\}\right]\right) \\
\sigma_{25}(s, t) & =-2 f \vartheta \ln (A)^{2}-2 f \chi \ln (A)^{2}\left(\sqrt{-\frac{\vartheta}{f}}\left[-\operatorname{coth}_{A}\{2 \sqrt{-\vartheta f} \phi\} \pm \sqrt{\Delta \Omega} \operatorname{csch}_{A}\{2 \sqrt{-\vartheta f} \phi\}\right]\right) \\
& -2 f^{2} \ln (A)^{2}\left(\sqrt{-\frac{\vartheta}{f}}\left[-\operatorname{coth}_{A}\{2 \sqrt{-\vartheta f} \phi\} \pm \sqrt{\Delta \Omega} \operatorname{csch}_{A}\{2 \sqrt{-\vartheta f} \phi\}\right]\right)^{2} .
\end{aligned}
$$

5) If $\vartheta=f$ and $\chi=0$, then singular new periodic solutions are presented by

$$
\begin{aligned}
\varepsilon_{6}(s, t) & =-\chi \ln (A)-2 f \ln (A)\left(\frac{1}{2}\left[\tan _{A}\left\{\frac{\vartheta}{2} \phi\right\}-\cot _{A}\left\{\frac{\vartheta}{2} \phi\right\}\right]\right), \\
\sigma_{26}(s, t) & =-2 f \vartheta \ln (A)^{2}+(-2) f \chi \ln (A)^{2}\left(\frac{1}{2}\left[\tan _{A}\left\{\frac{\vartheta}{2} \phi\right\}-\cot _{A}\left\{\frac{\vartheta}{2} \phi\right\}\right]\right) \\
& -2 f^{2} \ln (A)^{2}\left(\frac{1}{2}\left[\tan _{A}\left\{\frac{\vartheta}{2} \phi\right\}-\cot _{A}\left\{\frac{\vartheta}{2} \phi\right\}\right]\right)^{2} .
\end{aligned}
$$

6) If $\vartheta=-f$ and $\chi=0$, then dark and singular soliton new solutions are presented by

$$
\begin{aligned}
\varepsilon_{7}(s, t) & =-\chi \ln (A)-2 f \ln (A)\left(-\tanh _{A}(\vartheta \phi)\right), \\
\sigma_{27} & =-2 f \vartheta \ln (A)^{2}+(-2) f \chi \ln (A)^{2}\left(-\tanh _{A}(h \phi)\right)-2 f^{2} \ln (A)^{2}\left(-\tanh _{A}(\vartheta \phi)\right)^{2} .
\end{aligned}
$$

7) If $\chi^{2}=4 \vartheta f$, then rational new solution is presented by

$$
\begin{aligned}
\varepsilon_{8}(s, t) & =-\chi \ln (A)-2 f \ln (A)\left(-2 \vartheta \frac{\chi \phi \ln (A)+2}{\chi^{2} \phi \ln (A)}\right), \\
\sigma_{28}(s, t) & =-2 f \vartheta \ln (A)^{2}+(-2) f \chi \ln (A)^{2}\left(-2 \vartheta \frac{\chi \phi \ln (A)+2}{\chi^{2} \phi \ln (A)}\right)-2 f^{2} \ln (A)^{2}\left(-2 \vartheta \frac{\chi \phi \ln (A)+2}{\chi^{2} \phi \ln (A)}\right)^{2} .
\end{aligned}
$$

8) If $\chi=k, \vartheta=m k(m \neq 0)$ and $f=0$, then the rational solution is presented by

$$
\begin{aligned}
\varepsilon_{9}(s, t) & =-\chi \ln (A)-2 f \ln (A)\left(A^{k \phi}-m\right), \\
\sigma_{29}(s, t) & =-2 f \vartheta \ln (A)^{2}+(-2) f \chi \ln (A)^{2}\left(A^{k \phi}-m\right)-2 f^{2} \ln (A)^{2}\left(A^{k \phi}-m\right)^{2} .
\end{aligned}
$$


9) If $\chi=f=0$, then rational new solution is presented by

$$
\begin{aligned}
\varepsilon_{10}(s, t) & =-\chi \ln (A)-2 f \ln (A)(h \phi \ln (A)), \\
\sigma_{210}(s, t) & =-2 f \vartheta \ln (A)^{2}+(-2) f \chi \ln (A)^{2}(\vartheta \phi \ln (A))-2 f^{2} \ln (A)^{2}(\vartheta \phi \ln (A))^{2} .
\end{aligned}
$$

10) If $\chi=\vartheta=0$, then rational new solution is presented by

$$
\begin{aligned}
\varepsilon_{11}(s, t) & =-\chi \ln (A)-2 f \ln (A)\left(-\frac{1}{f \phi \ln (A)}\right) \\
\sigma_{211}(s, t) & =-2 f \vartheta \ln (A)^{2}+(-2) f \chi \ln (A)^{2}\left(-\frac{1}{f \phi \ln (A)}\right)-2 f^{2} \ln (A)^{2}\left(-\frac{1}{f \phi \ln (A)}\right)^{2} .
\end{aligned}
$$

11) If $\vartheta=0$, and $\chi \neq 0$, then dark like new solitons are presented by

$$
\begin{aligned}
\varepsilon_{12}(s, t) & =-\chi \ln (A)-2 f \ln (A)\left(-\frac{\Delta \chi}{f\left[\cosh _{A}\{\chi \phi\}-\sinh _{A}\{\chi \phi\}+\Delta\right]}\right), \\
\sigma_{212}(s, t) & =-2 f \vartheta \ln (A)^{2}+(-2) f \chi \ln (A)^{2}\left(-\frac{\Delta g}{f\left[\cosh _{A}\{\chi \phi\}-\sinh _{A}\{\chi \phi\}+\Delta\right]}\right) \\
& -2 f^{2} \ln (A)^{2}\left(-\frac{\Delta \chi}{f\left[\cosh _{A}\{\chi \phi\}-\sinh _{A}\{\chi \phi\}+\Delta\right]}\right)^{2} .
\end{aligned}
$$

12) If $\chi=k$, and $\vartheta=0$ and $f=m k(m \neq 0)$, then rational new solution is presented by

$$
\begin{aligned}
\varepsilon_{13}(s, t) & =-\chi \ln (A)-2 f(A)\left(\frac{\Delta A^{k \phi}}{\Omega-m \Delta A^{k \phi}}\right) \\
\sigma_{213}(s, t) & =-2 f \vartheta \ln (A)^{2}+(-2) f \chi \ln (A)^{2}\left(\frac{\Delta A^{k \phi}}{\Omega-m \Delta A^{k \phi}}\right)-2 f^{2} \ln (A)^{2}\left(\frac{\Delta A^{k \phi}}{\Omega-m \Delta A^{k \phi}}\right)^{2} .
\end{aligned}
$$

\section{Graphical representation of the solutions}

The magnetic flow graphics of the performed solutions are demonstrated in the illustrates by applying Mathematica. In Figs. 4-5, we produce some numerical simulations of $\varepsilon(s, t)$ and $\sigma_{2}(s, t)$ in $3 \mathrm{D}$ plots when $0 \leq s \leq 5$ and $0 \leq t \leq 5$.
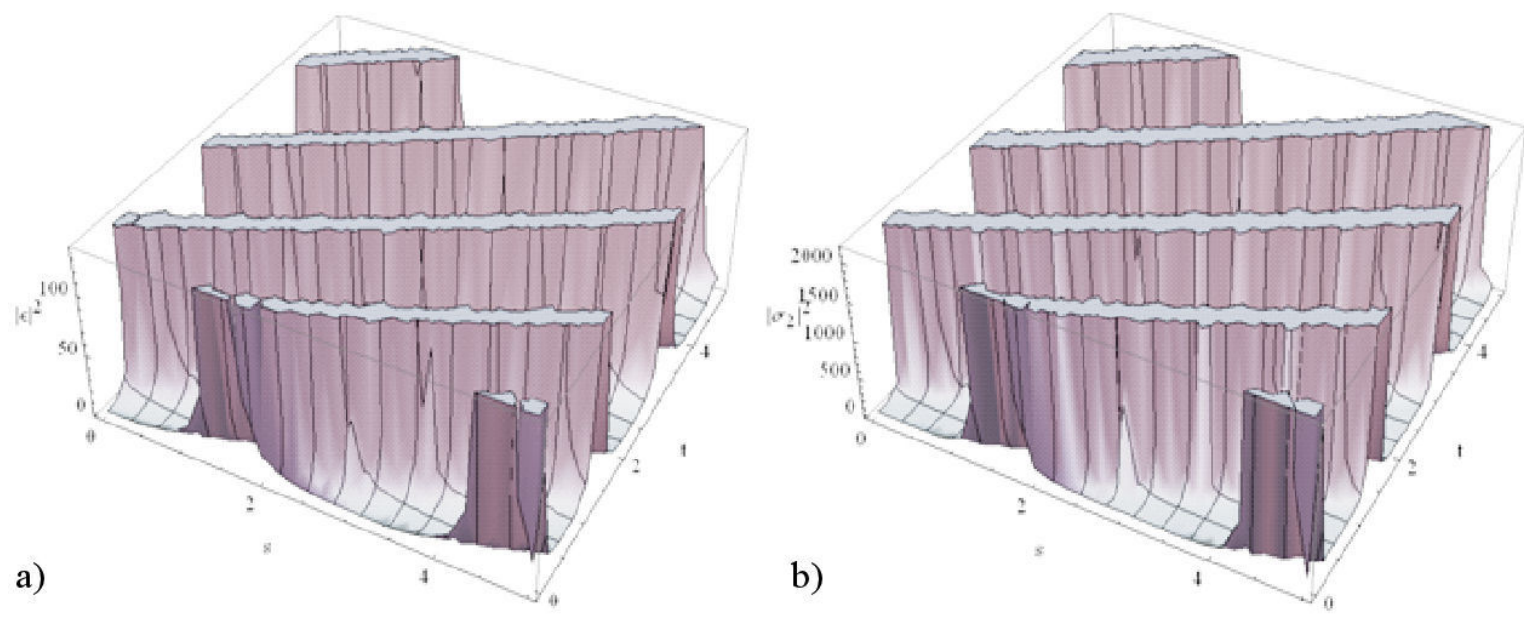

FIGURE 4. The magnetic flow graphics for the analitical solutions of the fractional (4.1) equations $(h=f=2, \chi=1)$ a) $\varepsilon_{1}(s, t)$, b) $\sigma_{21}(s, t)$. 

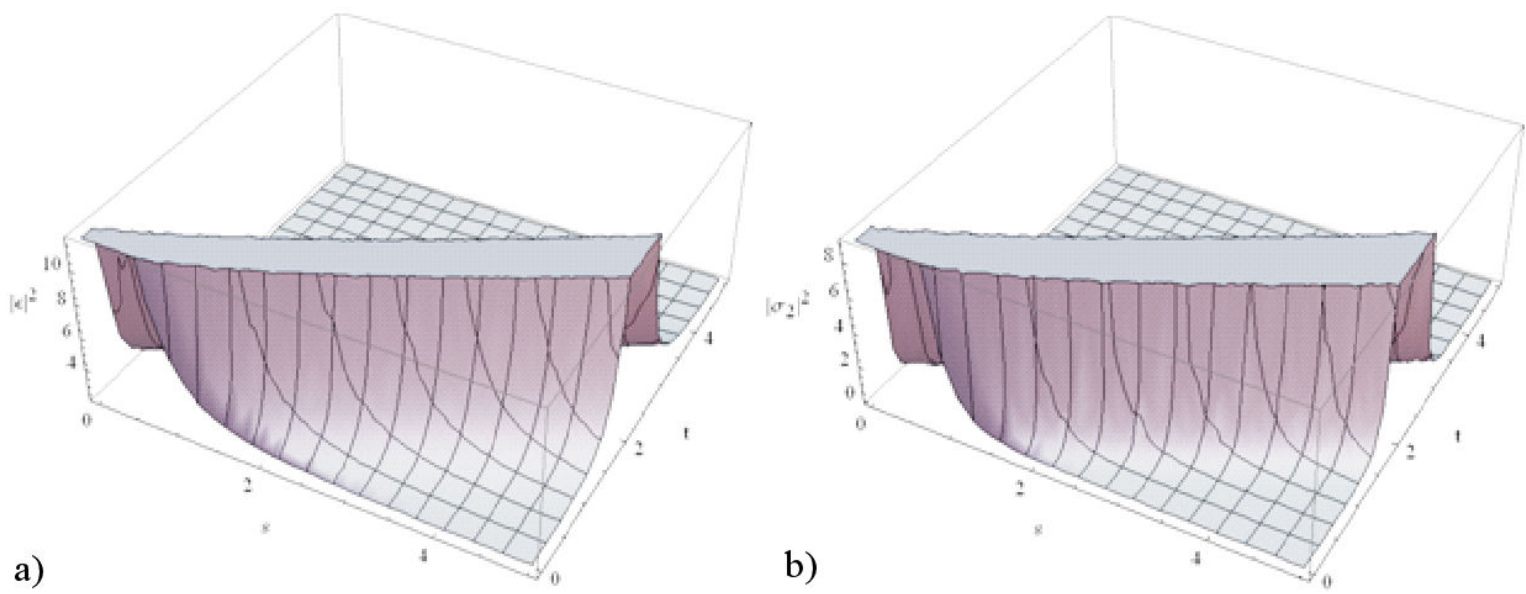

FIGURE 5. The magnetic flow graphics for the analitical solutions of the fractional (4.1) equations $(h=-2, f=1, \chi=0)$ a) $\varepsilon_{5}(s, t)$, b) $\sigma_{25}(s, t)$.

We displayed the some of solutions recovered for the presented fractional (4.1) equations by a conformable derivative operator. Besides, we showed 3D graphics for some of the solutions in Figs. 4-5. The above figures were drawn for $A=1.7, \eta=0.8, \Delta=\Omega=1$.

\section{Conclusion}

Geometrical models that describe a relativistic magnetic particle may be constructed using the geometrical scalars associated with the embedding of the particle worldline in Heisenberg spacetime as building blocks for the action. In this investigation, we consider a new theory of optical magnetic spherical antiferromagnetic spin of timelike spherical magnetic flows of the t-magnetic particle by the spherical Sitter frame in de Sitter space. Also, the extended direct algebraic method is used to find new soliton solutions of the fractional (4.1) equations in de Sitter space. Many partial differential equations are proceeding in mathematical physics and engineering modeling applications [31-46].

We express that the suggested approach is applicable to investigate the plenty of illustrations based on engineering and science.

1. A. M. Smith Appl. Opt. 17 (1978) 52. https://doi.org/ $10.1364 / \mathrm{AO} .17 .000052$

2. J. N. Ross, Opt. Quantum Electron. 16 (1984) 455. https: //doi.org/10.1007/BF00619638

3. A. Tomita and Y. Chiao, Phys. Rev. Lett. 57 (1986) 937. https://doi.org/10.1103/PhysRevLett.57. 937

4. R. Y. Chiao and Y. S. Wu, Phys. Rev. Lett. 57 (1986) 933. https://doi.org/10.1103/PhysRevLett.57. 933

5. F D. M. Haldane Optics Lett. 11 (1986) 730. https: / / doi. org/10.1364/OL.11.000730

6. T. Körpınar and R. C. Demirkol, J. Mod. Optics. 66 (2019) 857. https://doi.org/10.1080/09500340.2019. 1579930

7. T. Körpınar, R. C. Demirkol and Z. Körpınar, Eur. Phys. J. D 73 (2019) 203. https://doi.org/10.1140/epjd/ e2019-100250-7

8. T. Körpınar, R. C. Demirkol and Z. Körpınar, Int. J. Geom. Methods Mod. Phys. 16 (2019) 1950117.

9. T. Körpınar, R. C. Demirkol and Z. Körpınar, Rev. Mex. Fis 65 (2019) 626. https://doi.org/10.31349/ RevMexFis.65.626
10. T. Körpınar and R. C. Demirkol, Optik 200 (2020) 163334. https://doi.org/10.1016/j.ijleo.2019. 163334

11. G. L. Lamb, J. Math. Phys. 18 (1977) 1654. https: //doi. org/10.1063/1.523453

12. S Murugesh and R. Balakrishnan, Phys. Lett. A 290 (2001) 81. https://doi.org/10.1016/S0375-9601(01) $00632-6$

13. M. V Berry, Proc. R. Soc. Lond. A. Math. Phys. Sci. 392 (1984) 45.https://doi.org/10.1098/rspa.1984.0023

14. X S Fang and Z. Q Lin, IEEE Trans. Microwave TheorY. Tech. MTT 33 (1985) 1150. DOI: $10.1109 /$ TMTT . 1985. 1133187

15. J. N Ross, Opt. Quantum Electron. 16 (1984) 455.

16. A. TomitA. and R. Y. Chiao, Phys. Rev. Lett. 57 (1986) 937.

17. F Wassmann and A. Ankiewicz, Applied Optics 37 (1998) 18. https://doi.org/10.1364/AO.37.003902

18. R. Balakrishnan, R. Bishop and R. Dandoloff, Phys. Rev. Lett. 64 (1990) 2107. https://doi.org/10.1103/ PhysRevLett. 64.2107

19. R. Balakrishnan, R. Bishop and R. Dandoloff, Phys. Rev. B 47 (1993) 3108. https://doi.org/10.1103/PhysRevB. 47.5438 
20. R. Balakrishnan and R. Dandoloff, Phys. Lett. A 260 (1999) 62. https://doi.org/10.1016/s0375-9601(99) $00492-2$

21. T. Körpınar, Acta Scientiarum Technology 37 (2015) 245. https://doi.org/10.4025/actascitechnol. v37i2.17368

22. T. Körpinar and R. C. Demirkol, Int. J. Geom. Methods Mod. Phys. 15 (2018) 1850020. https://doi.org/10.1142/ S0219887818500202

23. T. Körpinar and R. C. Demirkol, Int. J. Geom. Methods Mod. Phys. 15 (2018) 1850184. https: / /doi .org/10.1142/ S0219887818501840

24. I. Podlubny, Fractional Differential Equations (Academic Press, San Diego) (1998).

25. A. A. Kilbas, H. M. SrivastavA. and J. J. Trujillo, TheorY. and Applications of Fractional Differential Equations (Elsevier, Amsterdam) (2006).

26. J. Sabatier, O. P Agrawal and J. A. T. Machado. Advances in Fractional Calculus (SpringeR. Dordrecht) (2007).

27. F. Mainardi, Fractional Calculus and Waves in LineaR. Viscoelasticity: An Introduction to Mathematical Models (World Scientific, Singapore) (2010).

28. X J. Yang, General Fractional Derivatives: Theory, Methods and Applications (CRC Press, New York) (2019).

29. R. Khalil, M. Al Horani, A. Yousef and M. Sababheh, J. Comput. Appl. Math. 264 (2014) 65. https://doi.org/10. $1016 /$ j.cam.2014.01.002

30. M. Eslamin and H. Rezazadeh Calcolo 53 (2016) 475. DOI : https://doi.org/10.31349/RevMexFis.66.297

31. Y. Çenesiz, D. Baleanu, A. Kurt and O. Tasbozan, Waves Random Complex Media 27 (2017) 103. https://doi.org/ $10.1080 / 17455030.2016 .1205237$

32. T. Körpınar, R. C. Demirkol and Z. Körpınar, Optik - International Journal for light and Electron Optics 238 (2021) 166403. https://doi.org/10.1142/S0219887821501851
33. T. Körpinar and R. C. Demirkol Ukrainian Mathematical Journal, 72 (2021) 1267. https://doi.org/10.1007/ s11253-020-01853-3

34. T. Körpınar, Adv. Appl. Clifford Algebras, 28 (2018) 9. https://doi.org/10.1007/s00006-018-0900-9

35. T. Körpınar, ZeitschrifT. für Naturforschung A. 70 (2015) 477. https://doi.org/10.1515/zna-2015-0044

36. M. Yeneroğlu, T. Körpınar, Journal of Advanced Physics. 7 (2018) 292. https://doi.org/10.1166/jap.2018. 1425

37. T. Körpınar, R. C. Demirkol, Z. Körpınar, V. Asil Rev. Mex. Fis 66 (2020) 431. https://doi.org/10.31349/ RevMexFis.66.431

38. T. Körpınar, R. C. Demirkol, Z. Körpınar, V. Asil Optik, 217 (2020) 164561.

39. X.- J. Yang, J. A. T. Machado Mathematical Methods in the Applied Sciences 42 (2019) 7539 https://doi.org/10. 31349/RevMexFis.67.452

40. T. Körpınar, Y. Ünlütürk AIMS Mathematics 5 (2020) 1025. DOI: $10.3934 /$ math.2020071

41. T. Körpınar, R. C. Demirkol, Optik - International Journal for light. and Electron Optics 200 (2020) 163334. https: //doi.org/10.1016/j.ijleo.2019.163334

42. T. Körpınar, Optik - International Journal for light and Electron Optics 219 (2020) 165134. https://doi.org/10. 1016/j.ijleo.2020.165134

43. T. Köpınar, Int. J. Geom. Methods Mod. Phys. 16 (2019) 1950018.

44. T. Köpınar, Indian J. Phys 94 (2020) 1409-1421. https: //doi.org/10.1007/s12648-019-01596-3

45. T. Köpınar, R. C. Demirkol Math Meth Appl Sci. 42 (2019) 3069.

46. T. Köpınar Adv. Appl. Clifford Algebras, 28 (2018) 83. https : //doi.org/10.1007/s00006-018-0834-2 